\title{
Transplanted Adult Neural Stem Cells Express Sonic Hedgehog In Vivo and Suppress White Matter Neuroinflammation after Experimental Traumatic Brain Injury
}

\author{
Genevieve M. Sullivan ${ }^{1,2}$ and Regina C. Armstrong ${ }^{1,2}$ \\ ${ }^{1}$ Department of Anatomy, Physiology and Genetics, Uniformed Services University of the Health Sciences, 4301 Jones Bridge Road, \\ Bethesda, MD 20814, USA \\ ${ }^{2}$ Center for Neuroscience and Regenerative Medicine, Uniformed Services University of the Health Sciences, 4301 Jones Bridge Road, \\ Bethesda, MD 20814, USA
}

Correspondence should be addressed to Regina C. Armstrong; regina.armstrong@usuhs.edu

Received 6 January 2017; Revised 19 April 2017; Accepted 5 June 2017; Published 30 August 2017

Academic Editor: Pavla Jendelova

Copyright (C) 2017 Genevieve M. Sullivan and Regina C. Armstrong. This is an open access article distributed under the Creative Commons Attribution License, which permits unrestricted use, distribution, and reproduction in any medium, provided the original work is properly cited.

\begin{abstract}
Neural stem cells (NSCs) delivered intraventricularly may be therapeutic for diffuse white matter pathology after traumatic brain injury (TBI). To test this concept, NSCs isolated from adult mouse subventricular zone (SVZ) were transplanted into the lateral ventricle of adult mice at two weeks post-TBI followed by analysis at four weeks post-TBI. We examined sonic hedgehog (Shh) signaling as a candidate mechanism by which transplanted NSCs may regulate neuroregeneration and/or neuroinflammation responses of endogenous cells. Mouse fluorescent reporter lines were generated to enable in vivo genetic labeling of cells actively transcribing Shh or Gli1 after transplantation and/or TBI. Gli1 transcription is an effective readout for canonical Shh signaling. In $S h h^{\text {CreERT2 }}$;R26tdTomato mice, Shh was primarily expressed in neurons and was not upregulated in reactive astrocytes or microglia after TBI. Corroborating results in Gli1 ${ }^{C r e E R T 2}$;R26tdTomato mice demonstrated that Shh signaling was not upregulated in the corpus callosum, even after TBI or NSC transplantation. Transplanted NSCs expressed Shh in vivo but did not increase Gli1 labeling of host SVZ cells. Importantly, NSC transplantation significantly reduced reactive astrogliosis and microglial/macrophage activation in the corpus callosum after TBI. Therefore, intraventricular NSC transplantation after TBI significantly attenuated neuroinflammation, but did not activate host Shh signaling via Gli1 transcription.
\end{abstract}

\section{Introduction}

Transplantation of stem cells into specific locations in the central nervous system (CNS) parenchyma may be the most appropriate approach for testing restorative cell therapies for focal lesions, such as stroke or Parkinson's disease. However, diffuse injuries such as traumatic brain injury (TBI) may require approaches that can reach broader regions. Diffuse axonal injury in white matter tracts is the most common pathological feature of TBI $[1,2]$. TBI patients often suffer long-term disability [3], and no effective therapies are available to prevent the progression of white matter pathology $[4,5]$. Therefore, potential therapeutics must be developed to ameliorate the progression of pathology and promote repair following diffuse axonal injury from TBI.

NSCs transplanted within the ventricular system may interact dynamically with endogenous cells to attenuate neuroinflammation, which contributes to a cascade of secondary damage in white matter tracts. Neural stem cell (NSC) transplantation also has the potential to enhance regeneration of damaged tissue directly by replacing lost cells and/or indirectly through the synthesis of signaling factors that stimulate regenerative responses of endogenous cells in the host tissue [6]. Multipotent NSCs reside in the adult subventricular zone (SVZ) and are maintained by sonic hedgehog (Shh) signaling [7-10]. Isolated NSCs can synthesize Shh in vitro after 
differentiation [11]. Thus, NSC transplantation may be a means to increase Shh signaling. Shh signaling and NSC transplantation have each been reported to be immunomodulatory and to promote endogenous cell repair in the corpus callosum in experimental demyelination [12-15]. However, it is presently unknown whether intraventricular NSC transplantation can modulate neuroinflammation or neuroregeneration after TBI. Additionally, approaches to demonstrate in vivo activation of the Shh pathway have not been used to examine this potential mechanism of NSC interaction with endogenous cells.

To address these research gaps, we used a model of experimental TBI to examine the effects of intraventricular transplantation of adult NSCs on the endogenous NSC response in the SVZ and neuroinflammation in the corpus callosum. This impact model produces traumatic axonal injury in the white matter with degenerating axons dispersed among intact axons [16-18]. The white matter pathology is similar to diffuse axonal injury in TBI patients, but is primarily in the corpus callosum over the lateral ventricles. Importantly, axon damage and neuroinflammation (reactive astrocytes and microglia/macrophages) persist in the corpus callosum out to 6 weeks post-TBI [18]. This region of pathology in the corpus callosum is adjacent to the SVZ and thus facilitates analysis of the regenerative response of endogenous cells in the host SVZ $[16,17]$.

For potential clinical relevance to future autologous NSC transplantation strategies, our experimental design used intraventricular transplantation of a low dose of adult NSCs at two weeks after TBI. Analysis of the beneficial effects of NSC transplantation included both modulation of neuroinflammation and stimulation of SVZ neuroregeneration, which are each responses that can be regulated by Shh signaling. Shh ${ }^{\text {CreERT2 }}$ and Gli1 ${ }^{\text {CreERT2 }}$ mice were each crossed to reporter lines for inducible genetic in vivo labeling of cells synthesizing or responding to Shh, respectively. These studies broadly examine the potential for adult NSCs transplanted into the lateral ventricles to influence endogenous cells in the adjacent white matter and SVZ regions after TBI and specifically examine the regulatory mechanism of signaling through the Shh pathway.

\section{Materials and Methods}

Mice were housed and cared for in accordance with the guidelines of the National Institutes of Health and the Institutional Animal Care and Use Committee of the Uniformed Services University of the Health Sciences.

\subsection{Inducible Genetic In Vivo Labeling of Cells Synthesizing or} Responding to Shh. Mouse lines were purchased from Jackson Laboratories (Bar Harbor, ME) with information for each line listed in the supplemental materials Table S1 available online at https://doi.org/10.1155/2017/9342534. Mouse conditional driver lines, $\operatorname{Shh}^{\mathrm{CrEERT2}}\left(\operatorname{Shh}^{\mathrm{tm} 2(\mathrm{cre} / E R T 2) C j t} / J\right)$, or

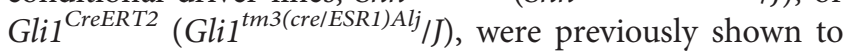
match mRNA transcript expression for inducible genetic labeling of cells expressing Shh [19] or cells responding to Shh signaling [20], respectively. Shh $h^{\text {CreERT2 }}$ mice exhibit reporter labeling consistent with Shh expression, and Shh expression was shown to be substantially reduced or eliminated in crosses to mice with floxed Shh alleles [21]. Gli1 ${ }^{\text {CreERT2 }}$ mice exhibit reporter labeling consistent with Gli1 expression detected by in situ hybridization [21], or using Gli1- $n$ LacZ mice [22]. The R26YFP reporter mice (B6.129X1$\mathrm{Gt}(R O S A) 26$ Sor $\left.^{\text {tm1 } 1(E Y F P) C o s} / \mathrm{J}\right)$ and the R26tdTomato reporter

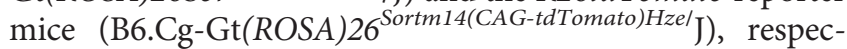
tively, contain the yellow fluorescent protein (YFP) or $t d T o-$ mato fluorescent protein (Tom) reporter genes downstream of a floxed stop codon $[23,24]$. The $R 26 \mathrm{mT} / \mathrm{mG}$ reporter mice (B6.129(Cg)-Gt(ROSA)26Sor tm4(ACTB-tdTomato,-EGFP)Luo $/ \mathrm{J})$ ubiquitously express membrane-targeted tdTomato $(m T)$ that switches to expression of membrane-targeted enhanced green fluorescent protein $(m G)$ after Cre-mediated recombination [25]. Gli1 ${ }^{\text {CreERT2 }}$ mice were crossed to either R26YFP or R26tdTomato mice. Shh ${ }^{\text {CreERT2 }}$ mice were crossed to either R26tdTomato or R26mT/mG mice. First-generation crosses were used for experimental procedures. To induce nuclear translocation for Cre-mediated recombination, $10 \mathrm{mg}$ of tamoxifen (TMX) (Sigma, St. Louis, MO) in corn oil was administered by oral gavage daily on days 2 and 3 after injury or NSC transplant [17].

2.2. Generation and Characterization of Adult NSCs. NSCs were isolated from the SVZ of 8-10-week-old mice and maintained as neurospheres, as in previously published protocols $[26,27]$. For NSC isolation, the dissected brain was cut into $1 \mathrm{~mm}$ coronal slices. The SVZ was dissected and enzymatically digested using the Neural Tissue Dissociation Kit (P) (Miltenyi Biotec Inc., San Diego, CA), followed by mechanical dissociation. Cells isolated from each mouse were plated in $10 \mathrm{ml}$ of mouse NeuroCult NSC Basal Medium (StemCell Technologies, Vancouver, BC, Canada) and supplemented with mouse NeuroCult NSC Proliferation Supplement (StemCell Technologies), $20 \mathrm{ng} / \mathrm{ml} \mathrm{rmEGF} \mathrm{(Life}$ Technologies, Grand Island, NY City, State), $10 \mathrm{ng} / \mathrm{ml}$ of rhFGF-b (Life Technologies), $2 \mu \mathrm{g} / \mathrm{ml}$ heparin (Life Technologies), and $100 \mathrm{U} / \mathrm{ml}$ pen/strep (Life Technologies). Cells were cultured at $37^{\circ} \mathrm{C}$ with $5 \% \mathrm{CO}_{2}$. Following the first passage, cells were dissociated to a single cell suspension in $0.025 \%$ Trypsin-EDTA (Invitrogen, Carlsbad, CA) and reseeded in culture medium at $1 \times 10^{5}$ cells $/ \mathrm{ml}$. Neurospheres from each isolation were cryopreserved at passage two in F12/DMEM (ThermoFisher) with 15\% dimethylsulfoxide (DMSO, ATCC). Cells were resurrected at least 2 weeks prior to transplantation and passaged at least once, but no more than five times, prior to transplantation (Figure S1). NSCs with constitutive green fluorescent protein (GFP) labeling (NSC-GFP) were generated from C57BL/6- $T g(U B C$ GFP)30Scha/J) mice. Shh ${ }^{\text {CreERT2 }} ; R 26 m T / m G$ mice were used to generate NSC-Shh cells. After TMX administration, cells with constitutive $\mathrm{mT}$ labeling convert to $\mathrm{mG}$ labeling if the Shh promoter is transcriptionally active in vivo.

To quantify the number of cells in primary neurosphere cultures, an aliquot of cells was taken from a single cell suspension of NSCs following extraction from a single mouse and after each passage. The aliquot of cells was diluted $1: 1$ in $0.4 \%$ trypan blue (Sigma) to determine cell viability based 


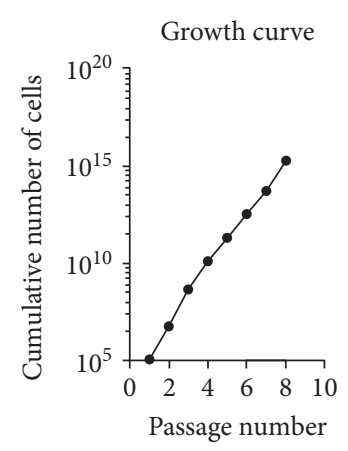

(a)

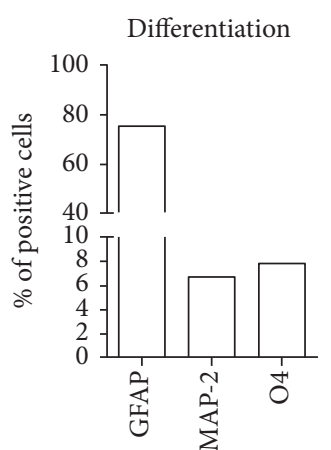

(b)

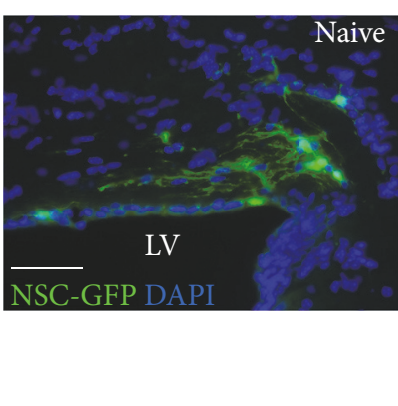

(c)

FIgURE 1: Adult NSC in vitro characterization and NSC-host response after transplantation. (a) In vitro growth curve of cumulative cells generated demonstrates proliferation. (b) In vitro confirmation of multipotency by quantification of differentiated cells immunolabeled for distinct lineage markers: astrocytes with GFAP, neurons with MAP-2, or oligodendrocytes with O4. (c) NSCs ubiquitously expressing green fluorescent protein (NSC-GFP) formed processes 1 week following transplantation into the parenchyma adjacent to the lateral ventricle $(\mathrm{LV})$. Sections were counterstained with 4',6-diamidino-2-phenylindole (DAPI, blue). Scale bar = 50 $\mu \mathrm{m}$ (c).

on trypan blue exclusion. The total number of cells (live + dead cells) were quantified using a phase contrast microscope and a hemocytometer. Starting at the first passage, the growth rate of cells was determined by dividing the total number of cells at the time of passage by the number of cells plated. The growth rate was then used to determine the cumulative number of cells following each passage to generate a growth curve. Proliferation through passage eight followed a linear growth curve with log phase amplification that is a characteristic of healthy stem cell self-renewal (Figure 1(a)).

NSCs were plated at densities of $4.8 \times 10^{4}$ cells $/ \mathrm{ml}$ for clonal analysis or 80,000 cells per coverslip and characterized as in previously published protocols [14]. Cultures were immunolabeled for markers of differentiation along the astroglial lineage (glial fibrillary acidic protein, GFAP; rabbit polyclonal, 1:100; DAKO, Carpinteria, CA), the neuronal lineage (microtubule associated protein-2, MAP-2; rabbit polyclonal, 1:200; Abcam, Cambridge, MA), and the oligodendroglial lineage $\mathrm{O} 4$ (mouse monoclonal, 1:10; [28]). Secondary antibodies used were goat anti-rabbit IgG conjugated with Alexa Fluor 488 (Life Technologies,) to detect GFAP and MAP-2 and goat anti-mouse IgM conjugated with FITC (Jackson ImmunoResearch, West Grove, PA) to detect O4. Neurospheres dissociated after passage five and cultured in differentiation medium demonstrated multipotency based on differentiation into astrocytes (GFAP), neurons (MAP-2), and oligodendrocyte lineage cells (O4) (Figures 1(b) and S1).

Adult NSCs isolated from mice ubiquitously expressing GFP (NSC-GFP) (Figure S1), and transplanted into naïve mice, demonstrated in vivo viability based on integration and extension of processes within the corpus callosum (Figure 1(c)).

\subsection{Lentiviral Transduction of Human Non-NSCs. Lentiviral} transduction was used to generate human embryonic kidney 293 (HEK293) cells expressing tdTomato (HEK-Tom). The pLVX-IRES-tdTomato vector (Clontech Laboratories, Mountain View, CA) was cotransfected with the viral core packaging construct PAX2 and the VSV-G envelope protein vector pCAG VSV-G into HEK293T cells (ATCC CRL-1573; Manassas, VA) to generate lentivirus for expression of tdTomato (Lenti-Tom), using previously published protocols [29]. Approximately, $70 \%$ of the Lenti-Tom-transduced HEK293T cells expressed the tdTomato reporter. HEK293 cells were authenticated as human by short tandem repeat profiling of an aliquot of the transfected cells using a 16-marker interspecies contamination test that resulted in $>80 \%$ match to human (IDEXX BioResearch, Columbia, MO (ATCC, Manassas, VA).

2.4. Surgical Procedures for TBI. TBI was produced in male (8-10 weeks old) Gli1 ${ }^{\text {CreERT2 }} ;$ R26tdTomato, Shh ${ }^{\text {CreERT2 }} ;$;26tdTomato or C57BL/6 J mice, as previously detailed $[16,17]$. Briefly, an Impact One $^{\mathrm{TM}}$ Stereotaxic Impactor device was aligned with a $3 \mathrm{~mm}$ flat tip to impact the skull at bregma using settings of $1.5 \mathrm{~mm}$ depth, $4.0 \mathrm{~m} / \mathrm{s}$ velocity (video recorded as $4.7 \mathrm{~m} / \mathrm{s}$ ), and $100 \mathrm{~ms}$ dwell time. These parameters have been shown to produce pathology in the corpus callosum with axon damage, astrogliosis, and microglial activation, similar to our previously characterized results [18]. Mice were excluded if the impact resulted in apnea $>30$ seconds or depressed fracture. Postsurgical data was recorded to characterize the injury across mouse lines. The righting reflex was significantly increased after TBI, compared to sham, in C57BL/6J mice (sham $0.56 \pm 0.12, n=5$; TBI $5.2 \pm$ $0.58, n=5 ; p<0.0001$ ), $\operatorname{Shh}^{\text {CreERT2 }} ; R 26$ tdTomato mice (sham $1.90 \pm 0.60, n=5$; TBI $7.20 \pm 1.91, n=10 ; p=0.0101)$, and Gli1 $^{\text {CreERT2 }}$;R26tdTomato (sham $2.11 \pm 0.31, \quad n=9$; TBI $5.43 \pm 0.719, n=8 ; p=0.0004)$. The righting reflex after TBI was not affected by the genetic background of these mouse lines $(p=0.3666)$. Surgical data includes only mice used for quantitative analysis in subsequent experiments. Fractures on the skull or contusions on the surface of the brain were not observed at the time of dissection. Sham surgery included anesthesia and scalp incision. After surgery, mice were randomly selected for vehicle or cell transplantation.

Two weeks prior to HEK-Tom cell transplantation, a cohort of mice received a controlled cortical impact (CCI). 
The CCI model of TBI was used to examine a more invasive injury with focal gray matter damage and blood-brain barrier disruption, as previously detailed [17]. Briefly, in male (8-10 weeks old) Gli1 ${ }_{\text {CreERT2 }}$ R26YFP or C57BL/6J mice, a craniotomy was performed to expose the dura mater. An Impact One Stereotaxic Impactor device (Leica Biosystems; Buffalo Grove, IL) was used to impact the dura mater of the cerebral cortex of the right hemisphere ( 0 bregma; $1.5 \mathrm{~mm}$ lateral). Settings used were $2 \mathrm{~mm}$ tip diameter, $1 \mathrm{~mm}$ depth, $1.5 \mathrm{~m} / \mathrm{s}$ velocity, and $100 \mathrm{~ms}$ dwell time. Mice used for CCI were Gli1 ${ }^{\mathrm{CrEERT2}}$;R26YFP (

2.5. Cell Transplantation into the Lateral Ventricle. Host mice for transplantation experiments were C57BL/ 6J, Shh ${ }_{\text {CreERT2 }}^{\text {;R26tdTomato, Gli1 }}{ }_{\text {CreERT2 }}^{\text {;R26tdTomato, or }}$ Gli1 ${ }^{\text {CreERT2 }}$;R26YFP. Briefly, a burr hole of approximately $1.0 \mathrm{~mm}$ diameter was drilled into the skull, unless a craniotomy for CCI was previously performed. A $10 \mu \mathrm{l}$ Hamilton gas tight syringe (Cat\# 7653-01; Hamilton Company, Reno, NV) with compression fitting adapters (Cat\# 55750-01; Hamilton Company) and a pulled glass micropipette (outer diameter $50 \mu \mathrm{m}$ ) was used to microinject $2 \mu \mathrm{l}$ of cell suspension $\left(2.5 \times 10^{4}\right.$ cells/ul $)$ or F12/DMEM vehicle (ThermoFisher) into the lateral ventricle $(1.0 \mathrm{~mm}$ to the right of bregma and $-1.75 \mathrm{~mm}$ deep). Transplanted cells were either NSC-GFP cells, NSC-Shh cells, or HEK-Tom cells.

2.6. Immunohistochemistry in Tissue Sections. Mice were perfused with $3 \%$ paraformaldehyde, and brains were processed as in previously published protocols [17]. Immunohistochemistry was performed to detect astrocytes expressing GFAP (rabbit polyclonal, 1 : 1000; DAKO), microglia/macrophage with CD11b (rat monoclonal, 1:100; AbCam), or T-lymphocytes with the pan T-cell marker CD3 (rabbit polyclonal, $1: 2000$; DAKO). Neurons were immunolabeled with neuronal nuclei marker NeuN (mouse monoclonal, 1:100; Sigma). Axons were identified by localization of neurofilaments (rabbit polyclonal neurofilament $\mathrm{H} ; 1: 500$; Encor Biotechnology, Gainesville, FL). Immunolabeling of nonphosphorylated neurofilaments (mouse monoclonal SMI-32; 1 : 1000; Covance, Gaithersburg, MD) was used to detect damaged axons [30]. YFP reporter expression was detected with an antibody against GFP (rabbit polyclonal, 1 : 1000; Life Technologies). In Gli1 ${ }_{\text {CreERT2 }}^{\text {;R26tdTomato or }}$ Gli1 $^{\text {CreERT2 }}$;R26YFP mice, goat anti-rabbit IgG conjugated with Alexa Fluor 488 (Life Technologies) was used to detect GFAP, CD3, or YFP and goat anti-rat IgG conjugated with Alexa Fluor 488 (Life Technologies) was used to detect CD11b. In C57BL/6J or Gli1 ${ }_{\text {CreERT2 }}$ R26tdTomato mice, goat anti-rat IgG conjugated with Alexa Fluor 555 (Life Technologies) was used to detect CD11b or donkey anti-rabbit IgG conjugated to $\mathrm{Cy} 3$ (Jackson Immunoresearch) was used to detect GFAP. Sections were counterstained with 4',6'-diamidino-2-phenylindole (DAPI; Sigma) then mounted with Vectashield (Vector Laboratories).

2.7. Quantification and Statistical Analysis. The number of mice used from each line is noted with the TBI surgical methods. Conditions were randomly assigned within each mouse cohort. All quantification was performed blinded to injury (TBI/sham) and transplantation (cells/vehicle) procedures. All tissue analysis was performed in coronal brain tissue sections. Analyses of the corpus callosum or SVZ used at least three sections per mouse distributed between approximately $+0.3 \mathrm{~mm}$ and $-0.2 \mathrm{~mm}$ relative to bregma. Analyses of NSC-Shh cells within the lateral and third ventricles used sections from approximately $-0.0 \mathrm{~mm}$ to $-1.6 \mathrm{~mm}$ relative to bregma, which were sampled at least every $300 \mu \mathrm{m}$ to reach a minimum of 50 NSC-Shh cells counted in each mouse. Images were collected using Spot RT3 digital camera (Diagnostic Instruments, Sterling Heights, MI). Spot Advanced software was used to measure the area of the corpus callosum and SVZ, as previously described [16, 17]. The area of GFAP or $\mathrm{CD} 11 \mathrm{~b}$ immunolabeling in the corpus callosum was quantified using Metamorph software (Molecular Devices, Downingtown, PA) to threshold pixels with intensities above background levels as previously detailed $[16,18]$. CD11b cells within the corpus callosum were also counted individually to differentiate activation stage as resting (fine processes), activated (hypertrophic with thickened processes), or ameboid (retracted processes) as previously characterized [16].

Prism 6.0 (GraphPad Software) was used for graphing and statistical analysis. Two-way analysis of variance (ANOVA) was performed to determine significant differences between NSC-transplanted and vehicle-injected cohorts across naïve, sham, and/or injured conditions. Significant differences between the righting response in sham and TBI cohorts of a single genotype were determined using the two tailed unpaired $t$-test, while one-way ANOVA was used to compare TBI conditions across the three genotypes. Values shown are mean \pm SEM. Statistical significance was determined as $p<0.05$.

\section{Results}

3.1. Induced Genetic In Vivo Labeling of Host Cells Expressing Shh. An impact model of TBI was used that produces axon damage in the corpus callosum, primarily above the lateral ventricles [16-18]. This injury localization facilitates analysis of potential effects from NSC cells transplanted into the adjacent ventricle in subsequent experiments. We first examined Shh expression following TBI using TMX administration to induce genetic labeling of cells actively transcribing Shh in vivo based on expression of the tdTomato reporter (ShhTom) in Shh ${ }^{\text {CreERT2 }}$;R26tdTomato mice (Figures 2(a) and 2(b)). In both TBI and sham mice, TMX administered after surgery induced Shh-Tom labeling predominantly in neuronal cell bodies, axons, and processes based on morphology and immunolabeling for NeuN (Figures 2(c), 2(d), 2(e), 2(f), 2(g), 2(h), 2(i), and S2). Shh-Tom neurons were distributed as previously characterized in adult mouse brain [31,32]. Shh-Tom neurons were present in the striatum, adjacent to the SVZ and extending processes to the SVZ (Figure 2(c)), and in the cerebral cortex, including regions under the site of impact (Figures 2(d) and 2(e)). Astrocytes, identified by expression of GFAP, did not colabel for Shh-Tom in the cortex (Figures 2(d) and 2(e)) or in the corpus callosum, even after TBI in regions with reactive astrocytes (Figures 2(f) 


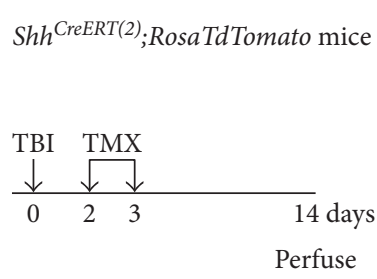

(a)

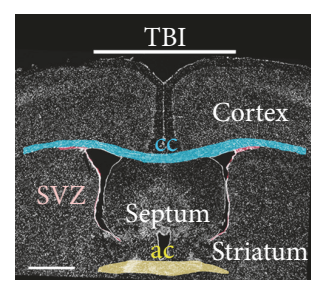

(b)

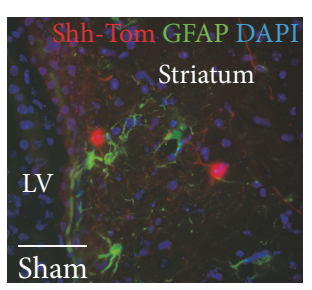

(c)

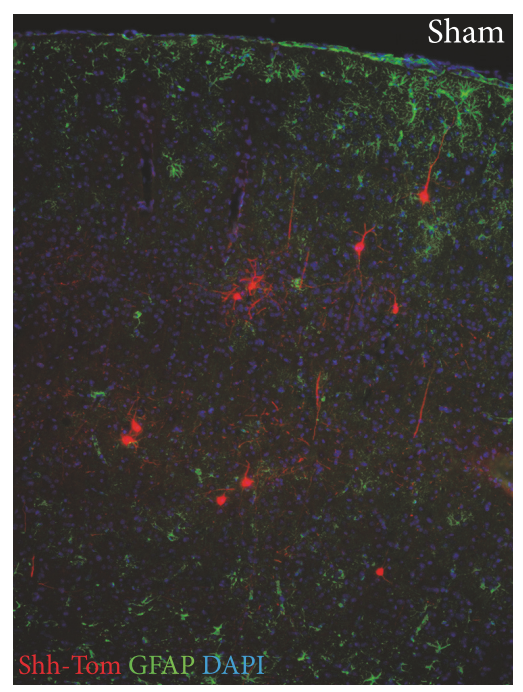

(d)

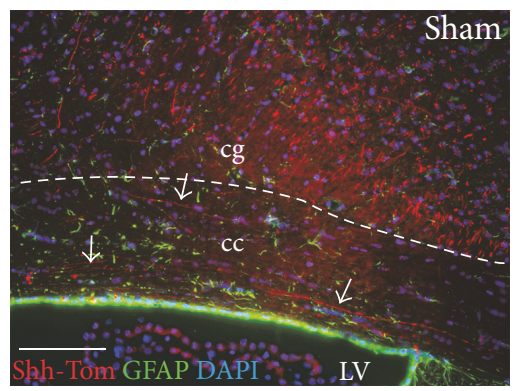

(f)

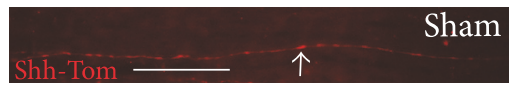

(h)

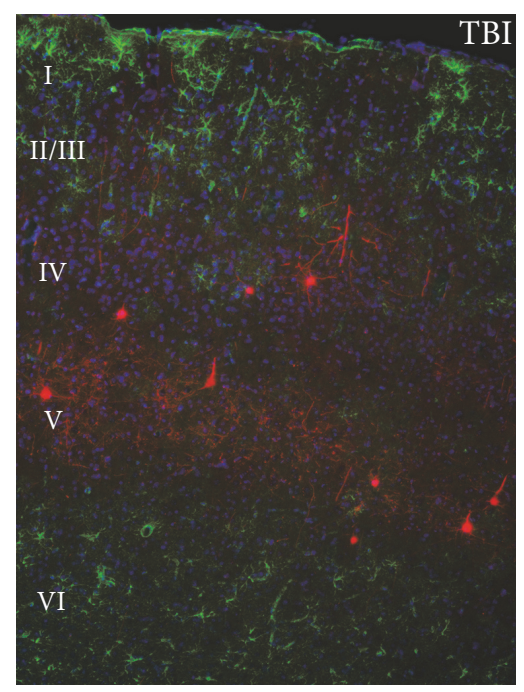

(e)

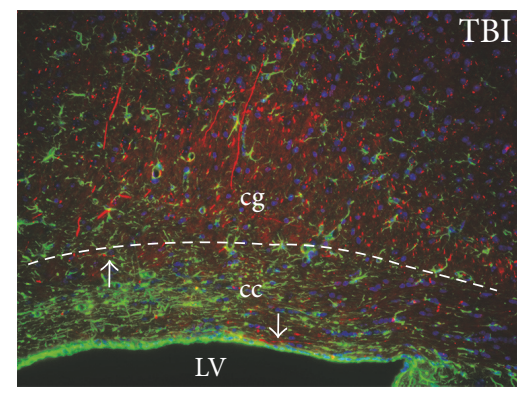

(g)

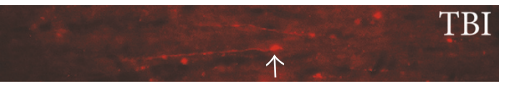

(i)

FIGURE 2: Induced genetic in vivo labeling to detect Shh expression following traumatic brain injury (TBI). (a) Shh ${ }^{\mathrm{CreERT2}}$;R26tdTomato mice received tamoxifen (TMX) 2 and 3 days after TBI or sham surgery and were perfused at 2 weeks postsurgery. (b) Diagram showing impact to the skull at bregma corresponded with the coronal level of the anterior commissure (ac). (c-g) Coronal sections with Shh transcription evident via the tdTomato reporter (Shh-Tom, red). GFAP immunolabeling (green) to detect astrocytic response to TBI. DAPI nuclear stain (blue) to identify cortical layers (I-VI). (c) Shh-Tom cells with neuronal morphology in the striatum near the SVZ and lateral ventricle (LV). Similar Shh-Tom cells in the striatum are colabeled with the neuronal marker NeuN (Figure S2). (d-e) Shh-Tom labeling in distributed cortical cells identified as neurons by morphology (d) and (e) colabeling with NeuN (Figure S2). GFAP immunolabeling in the cortex showed mild cortical astrogliosis after TBI (d-e). Shh-Tom cells did not have an astrocytic morphology or colabel for GFAP (d-e). (f-g) Shh-Tom labeling of axons in the cingulum (cg) and corpus callosum (cc) was similar in sham (f) and TBI (g) mice. After TBI, reactive astrocytes in the corpus callosum were not colabeled with Shh-Tom (g). (h-i) Shh-Tom axons were found in the corpus callosum but at a very low density. Normal variation of Shh-Tom labeling along the length of axons in a sham mouse (arrow; $(\mathrm{h})$ ). Consistent with axon damage after TBI, rare pathological swellings were observed in Shh-Tom-labeled axons (arrow; (i)). Scale bars $=1 \mathrm{~mm}$ (b), $100 \mu \mathrm{m}$ (c, d, f), $50 \mu \mathrm{m}$ (h). Representative images are shown from analysis of a cohort of sham $(n=3)$ and TBI $(n=4)$ mice.

and $2(\mathrm{~g})$ ). Indeed, Shh-Tom labeling of cell bodies was very rarely observed in the corpus callosum. Shh-Tom-labeled axons could be followed traversing the corpus callosum in both sham (Figures 2(f) and 2(h)) and TBI (Figures 2(g) and 2(i)) mice. Rare evidence of damage, that is, swelling and beading, in Shh-Tom-labeled axons was observed only 
after TBI (Figure 2(i)). These findings indicate that TBI does not stimulate Shh synthesis in reactive astrocytes and that the overall pattern of cells expressing Shh is similar between sham and TBI mice.

We next explored the potential for NSC transplantation to stimulate Shh signaling after TBI. Shh ${ }^{C r e E R T 2} ;$ R26tdTomato host mice had TBI or sham surgery and 2 weeks later received NSC-GFP cell transplant, or a vehicle injection, followed by perfusion for tissue analysis at 4 weeks postTBI or sham surgery (Figures 3(a) and 3(b)). Shh-Tom labeling near the lateral ventricles was similar to the labeling observed at two weeks after sham or TBI surgery (Figures 2(c), 3(c), and S2B). Primarily, Shh-Tom labeling was observed in cells with neuronal morphology, including cells in the striatum near the SVZ and axons extending toward the ventricle from septal regions (Figure 3(c)). ShhTom labeling was not altered by the close proximity of transplanted NSC-GFP cells (Figures 3(d), 3(e), 3(f), 3(g), and $3(\mathrm{~h}))$. Furthermore, with and without NSC-GFP transplantation, Shh-Tom labeling of axons appeared similar even in the corpus callosum and cingulum, which are regions with axon damage in this TBI model (Figure S3). Finally, near the injection tract, reactive astrocytes did not exhibit Shh-Tom labeling (data not shown). Overall, neither TBI nor NSCGFP transplantation markedly changed the pattern of host cells expressing Shh.

\subsection{Induced Genetic In Vivo Labeling Demonstrates NSCs} Expressing Shh after Transplantation. NSCs have been shown to synthesize Shh following in vitro differentiation [11]. Therefore, we examined the potential for NSCs to express Shh after transplantation. SVZ NSCs were cultured from $\mathrm{Shh}^{\mathrm{CreERT2}}$;R26mT/mG mice (NSC-Shh cells) to be able to detect transplanted NSCs with and without Shh expression in vivo. In NSC-Shh cells actively transcribing Shh, TMX administration induces a switch of membrane labeling from tdTomato (red) to GFP (green). Overlap appears as yellow in cells that had preexisting membranes labeled by tdTomato and then have begun to express GFP. Two weeks post-TBI or sham surgery, NSC-Shh cells were transplanted into the lateral ventricle of C57BL/6J mice and TMX was delivered on days 2 and 3 after transplantation (Figures 4(a) and 4(b)). After injection into the lateral ventricle, NSC-Shh cells distributed in both the lateral ventricles and the third ventricle, which were analyzed as sites near and remote from the injury, respectively (Figure 4(c)). TMX induced GFP expression indicative of active Shh expression in vivo (Figure $4(\mathrm{~d})$ ). In sham mice, approximately $60-80 \%$ of the transplanted NSC-Shh cells exhibited GFP labeling in either the lateral ventricle or the third ventricle (Figures 4(d), 4(e), 4(e'), and $\left.4\left(e^{\prime \prime}\right)\right)$. Remarkably, TBI resulted in a significant reduction in the proportion of NSC-Shh cells labeled with GFP (Figures 4(d), 4(f), 4(f), and 4(f')). This suppression of Shh expression in NSC-Shh cells after TBI was only found among cells in the lateral ventricle region, indicating a specific NSC response based on proximity to the injury (Figure $4(\mathrm{~d})$ ).

The majority of NSC-Shh cells in the ventricles had a rounded immature morphology (Figures 4(e) and 4(f')). For comparison with the intraventricular environment,
NSC-Shh cells are shown after transplantation directly into white matter adjacent to the ventricles (Figures $4(\mathrm{~g}), 4\left(\mathrm{~g}^{\prime}\right)$, and $\left.4\left(g^{\prime \prime}\right)\right)$. NSC-Shh cells in the parenchyma often elaborated processes and were labeled with GFP, indicating active Shh expression in vivo (Figures $4(\mathrm{~g}), 4\left(\mathrm{~g}^{\prime}\right)$, and $\left.4\left(\mathrm{~g}^{\prime \prime}\right)\right)$. In NSC-Shh cells that did not express Shh, the membranes of the cell bodies and extended processes continue to be labeled with tdTomato. In NSC-Shh cells that expressed Shh in vivo, the switch to GFP expression results in only GFP labeling of new membranes synthesized as cell processes form. In contrast, cell bodies appear yellow from the overlap of preexisting tdTomato-labeled membrane and the subsequent GFP membrane labeling.

3.3. Analysis of NSC Transplantation on Shh Signaling in Host SVZ Cells. Shh signaling regulates NSC maintenance in the SVZ of adult mice $[10,21]$. Therefore, we next examined whether NSCs transplanted into the lateral ventricle would increase Shh signaling in endogenous cells in the adjacent SVZ after TBI (Figures 5(a), 5(b), and 5(c)). NSC-GFP cells (Figure S1) were transplanted into Gli1 ${ }^{\mathrm{CrEERT2}}$; R26tdTomato mice. TMX was administered on days 2 and 3 posttransplantation to detect changes in the population of endogenous cells responding to Shh signaling, based on induced genetic labeling with tdTomato (Glil-Tom). Within the lateral ventricle, NSC-GFP cells were typically adhered to the walls of the ventricle and nestled within the choroid plexus (Figures 5 and S4). In comparison with naïve mice (Figure 5(d)), the endogenous Gli1-Tom population in the SVZ was not altered by the microinjection technique or NSC-GFP transplantation in either sham mice (Figure 5(e)) or after TBI (Figure 5(f)). The overall pattern of Gli1-Tom labeling in the areas adjacent to the lateral ventricles was also similar across conditions after vehicle or NSC-GFP injection (Figure S4). In addition to the SVZ, Gli1-Tom labeling was observed in distributed cells in the choroid plexus within the lateral ventricles (Figures 5 and S4). Gli1-Tom cells were only very rarely observed in the corpus callosum, even after TBI (Figures 5 and S4).

3.4. Analysis of NSC Transplantation on Neuroinflammation and Shh Signaling in the Corpus Callosum. We next evaluated the effect of NSC transplantation on TBI-induced pathology (Figure 6). This TBI model produces well-characterized axonal damage and neuroinflammation in the corpus callosum $[16,18]$. As shown in these prior studies, axon damage at longer time points after injury may not be adequately quantified based on $\beta$-amyloid precursor protein accumulation. Therefore, axon damage was detected using SMI-32 antibody, which recognizes nonphosphorylated neurofilaments [30]. Analysis of damaged axons focused on the cingulum, under the impact site, to image axons in cross-section for more accurate quantification (Figures 6(b), 6(c), and 6(d)). This analysis was performed using the Gli1 ${ }^{\text {CreERT2 }}$;R26tdTomato as analyzed in Figure 5. TBI significantly increased axon damage when compared to sham mice. However, NSC transplantation did not have a significant effect on reducing axon damage. 


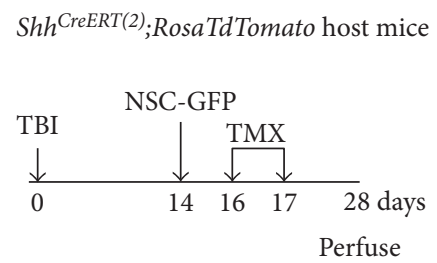

(a)

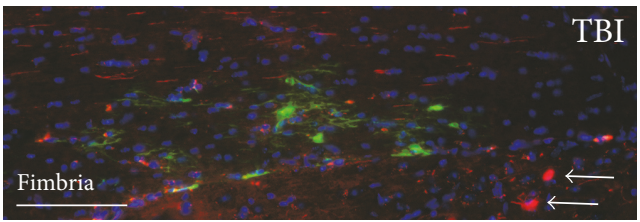

(d)

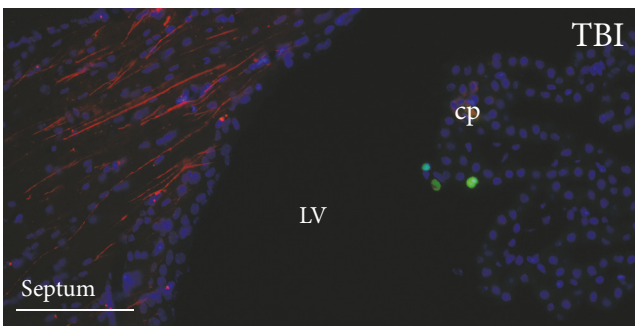

(e)

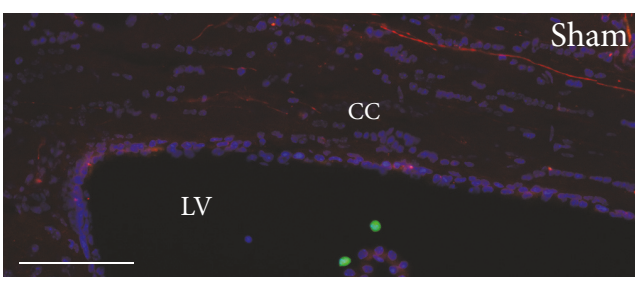

(f)

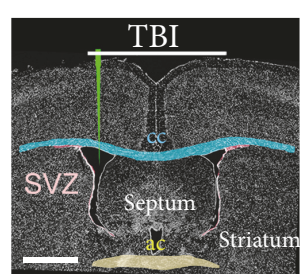

(b)

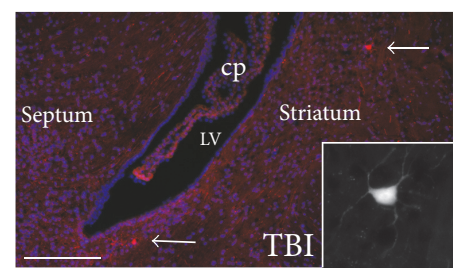

(c)

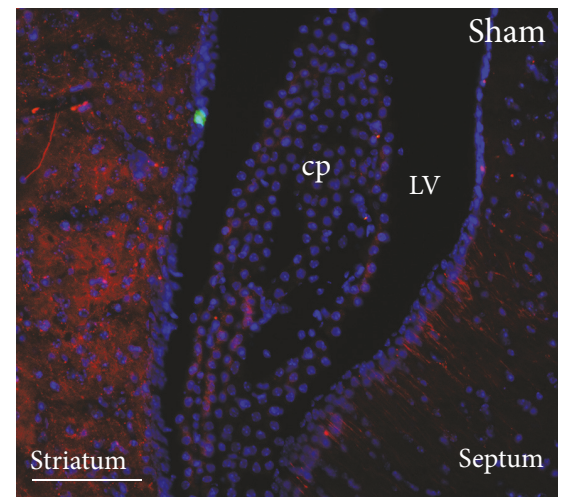

(g)

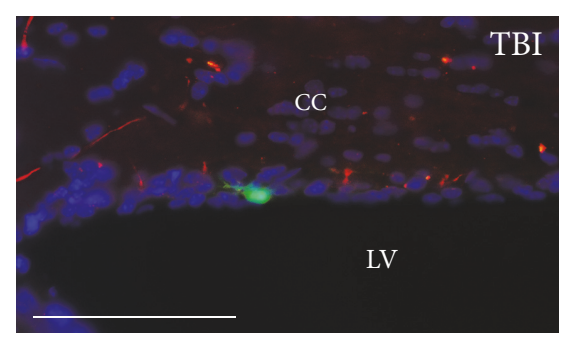

(h)

FIGURE 3: Induced genetic in vivo labeling of host cells expressing Shh after NSC transplantation into mice with traumatic brain injury (TBI). Timeline (a) and diagram (b) for experiments in $S_{h}{ }^{C r e E R T 2} ; R 26 t d$ Tomato host mice. Two weeks after TBI or sham surgery mice received an injection into the lateral ventricle (LV) of either NSCs constitutively expressing green fluorescent protein (NSC-GFP cells), or vehicle. To induce genetic labeling of host cells expressing Shh (Shh-Tom cells), TMX was administered on days 2 and 3 after injection of NSCs/ vehicle. Mice were perfused 4 weeks after TBI/sham surgery. (c-h) Coronal sections showing Shh-Tom labeling (red) with DAPI nuclear stain (blue) and transplanted NSC-GFP cells (green). Shh-Tom labeling in cell bodies ((c-d) arrows) and axons (e-h). Inset (c) shows neuronal morphology of Shh-Tom cells (arrows) in the striatum with example from upper arrow. Injections occasionally missed the LV resulting in NCS-GFP transplantation into the adjacent tissue and serve to show the potential to elaborate extensive processes is dependent on the transplant site (d). NSC-GFP cells transplanted directly into the LV grew as spheres (e, f) and were often observed nestled in the choroid plexus (cp), or extended only short processes when adhered to the walls of the LV (g, h). Scale bars $=1 \mathrm{~mm}(\mathrm{~b})$, $200 \mu \mathrm{m}(\mathrm{c}), 100 \mu \mathrm{m}(\mathrm{d}-\mathrm{g}), 50 \mu \mathrm{m}$ (h). Representative images are shown from analysis of a cohort of sham + NSC-GFP $(n=2)$, TBI + vehicle $(n=3)$, and TBI + NSC-GFP $(n=3)$ mice.

Shh signaling may also modulate neuroinflammation, that is, astrocyte and microglial reactivity, in the corpus callosum, as has been reported after experimental demyelination [13]. Therefore, we examined the potential for transplanted NSC-GFP cells to attenuate TBI-induced neuroinflammation in the corpus callosum adjacent to the lateral ventricles (Figures 6(e), 6(f), 6(g), 6(e'), 6(f), and 6(g')). TBI resulted in astrogliosis and macrophage/microglia activation in the corpus callosum that persisted at 4 weeks post-TBI (Figures 6(f) and 6(g)), as compared to sham mice (Figure 6(e)). Quantification of GFAP immunoreactivity showed an increase in astrocyte reactivity after TBI that was significantly reduced by NSC-GFP transplantation (Figure 6(e')). Quantification of CD11b immunoreactivity also demonstrated increased activation of microglia/macrophages after TBI that was significantly reduced by NSC-GFP transplantation (Figure 6(f)). Furthermore, CD11b-immunolabeled cells with an activated morphology (hypertrophic processes) were also increased after TBI and reduced by NSC-GFP transplantation (Figure 6(g')). These results demonstrate that NSC-GFP transplantation suppressed white matter neuroinflammation induced by TBI (Figures 6(e'), 6(f), and 6(g')).

Only very rare Gli1-Tom-labeled cells were detected in the corpus callosum of these Gli1 ${ }^{\text {CreERT2}}$;R26tdTomato mice 
C57BL/6J host mice

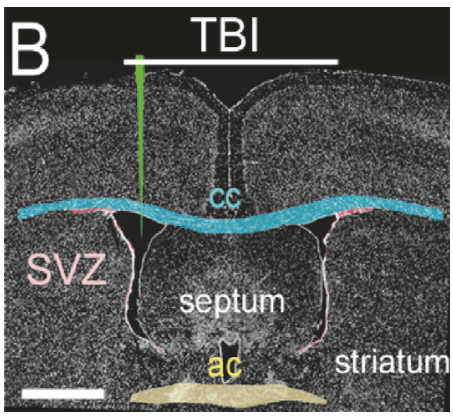

(a)

(b)

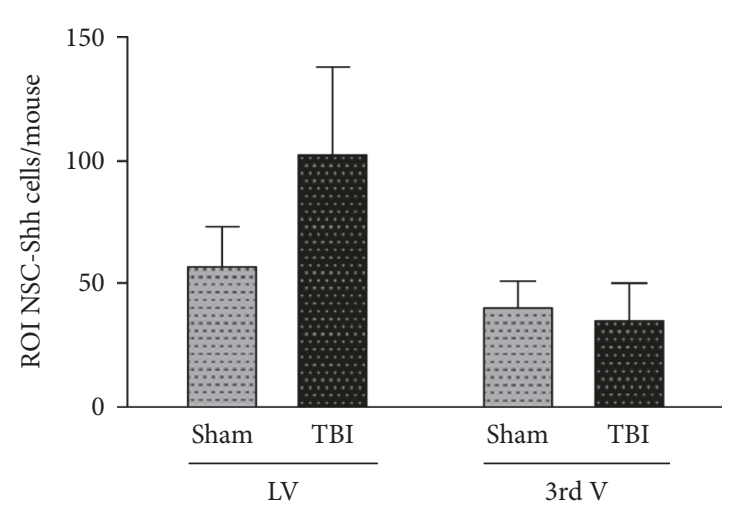

(c)

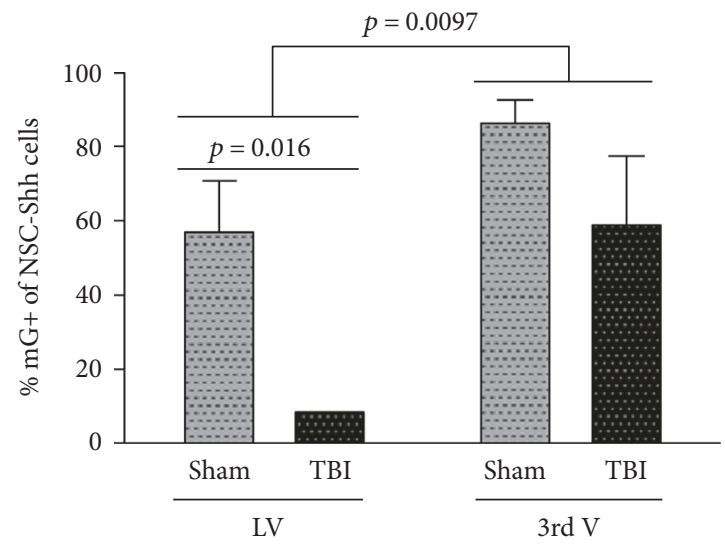

(d)

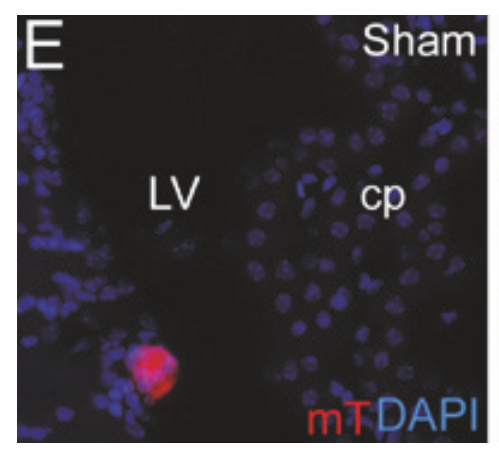

(e)

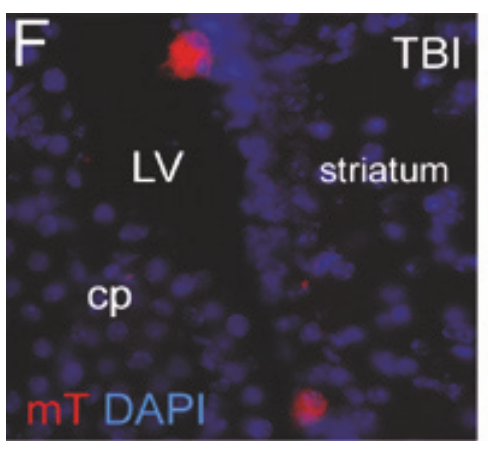

(f)

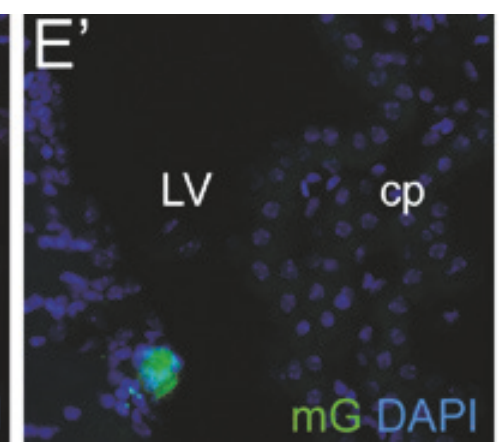

(e')

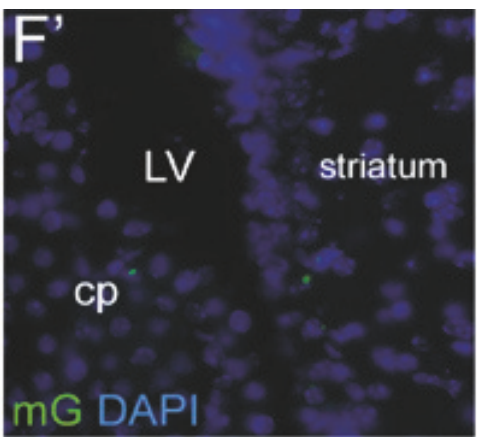

(f)

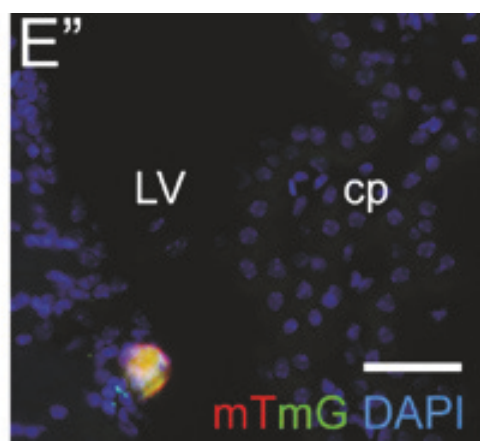

(e")

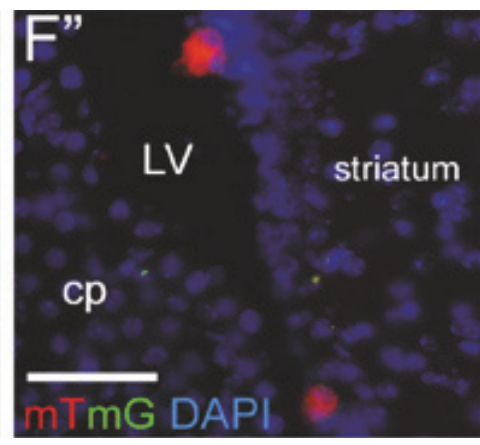

(f')

Figure 4: Continued. 


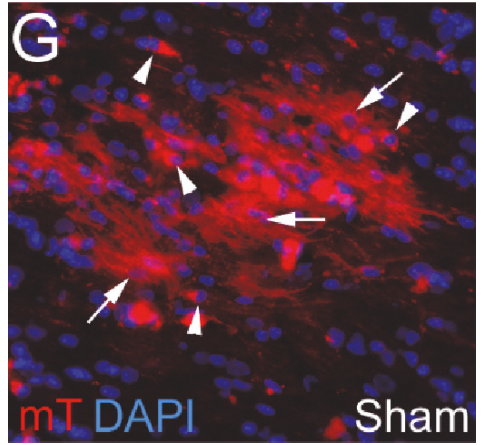

(g)

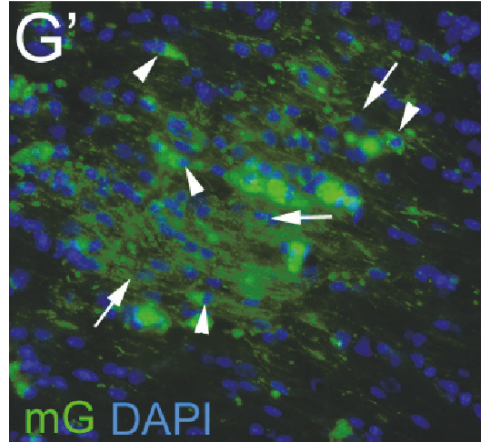

$\left(g^{\prime}\right)$

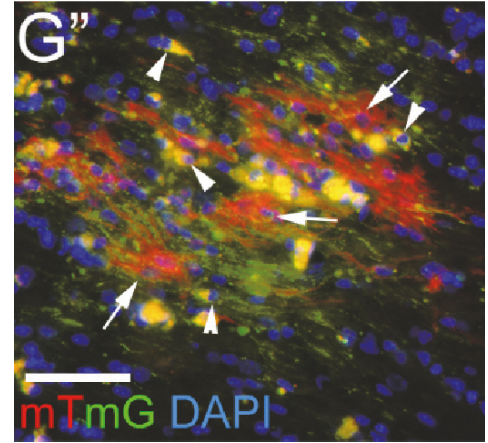

(g")

FIGURE 4: Induced genetic in vivo labeling of NSCs expressing Shh after transplantation. Timeline (a) and diagram (b) for host C57BL/6J mice that had TBI or sham surgery and 2 weeks later received an injection into the lateral ventricle (LV) of NSCs isolated from $\mathrm{Shh}^{\mathrm{CreERT2}} ; \mathrm{R} 26 \mathrm{mT} / \mathrm{mG}$ mice (NSC-Shh cells). Tamoxifen (TMX) was administered to induce a genetic switch from constitutive mT (red) to $\mathrm{mG}$ (green) fluorescence in transplanted NSC-Shh cells expressing Sh. Membranes that were already labeled by constitutive mT and then begin to express $\mathrm{mG}$ after TMX may exhibit both $\mathrm{mT}$ and $\mathrm{mG}$, so that colabeled membranes appear yellow. Mice were perfused at 4 weeks after TBI or sham surgery. (c) Transplanted NSC-Shh cell distribution within the lateral ventricles and third ventricle. (d) Proportion of transplanted NSC-Shh cells that exhibited expression of $\mathrm{mG}$, indicating in vivo Shh expression. In TBI mice, mG labeling was significantly reduced near the site of injury in the LV but not in the third ventricle. (e-f") Coronal sections showing transplanted NSC-Shh cells and DAPI nuclear stain (blue) to visualize the choroid plexus (cp) and wall of the LV. Sham mice shown with NSC-Shh cells in the LV that expressed both the $\mathrm{mT}$ (red) and $\mathrm{mG}$ (green) reporters, with overlap (yellow), indicating in vivo expression of Shh after transplantation (e-e"). TBI mice shown with two clusters of NSC-Shh cells in the LV that express constitutive mT (red) but not Shh-driven $\mathrm{mG}$ (green) (f-f'). Shh-driven $\mathrm{mG}$ expression was more evident in cells transplanted into the parenchyma, which extended processes ((g-g"), fimbria). After TMX induction, membranes appear green in newly elaborated processes, but appear yellow in cell bodies where $\mathrm{mT}$ and $\mathrm{mG}$ overlap (arrowheads). Other transplanted cells and processes are labeled by only $\mathrm{mT}$ expression (arrows), indicating lack of Shh expression or inefficiency of Cre recombination in vivo. Scale bars $=1 \mathrm{~mm}$ (b), $50 \mu \mathrm{m}(\mathrm{d}, \mathrm{f}), 25 \mu \mathrm{m}(\mathrm{e})$. ROI = region of interest quantified for designated rostrocaudal extents of the lateral ventricles and the third ventricle (see Methods). Quantification included analysis of a cohort of sham $(n=5)$ and TBI $(n=5)$ mice.

(Figures 5(d), 5(e), 5(f), 5(g), 5(h), and 5(i)). This result demonstrates that the TBI pathology did not activate canonical Shh signaling in reactive astrocytes or microglia. Furthermore, the immunomodulatory effect observed from NSC transplantation is likely mediated through pathways that do not activate canonical Shh signaling.

\subsection{Activation of Shh Responsive Cells in Host SVZ after} Invasive Brain Injury and HEK Cell Transplantation. To more accurately interpret the lack of changes in Gli1-Tom labeling among endogenous cells after TBI and/or NSC transplantation, a further set of experiments was designed as a positive control to elicit induced genetic in vivo labeling of Shh responsive cells in the SVZ. Shh signaling has been shown to increase after invasive injury, which involves a robust neuroinflammatory response $[17,33]$. Therefore, we predicted that a strong immune rejection response would be observed after transplantation of HEK cells, which are human non-NSC cells. And, using controlled cortical impact (CCI), which is a more invasive form of TBI, would be more permissive to an immune response due to cortical tissue damage and breakdown of the blood-brain barrier. At 2 weeks after CCI, HEK cells expressing the tdTomato reporter (HEK-Tom cells) were transplanted into $\mathrm{Gli1}^{\mathrm{CreERT2}}$ :R26YFP mice (Figure 7(a)). Mice were administered TMX on days 2 and 3 after the HEK-Tom cell transplantation. Immunohistochemistry for CD3, a pan T-cell marker, showed T-lymphocyte infiltration in regions surrounding HEK-Tom cells as early as 1 week following transplantation
(Figure 7(b)). This immune reaction to HEK-Tom cells did not require significant tissue damage, since it is present in sham mice that only received a craniotomy (Figure $7(b)$ ). CCI resulted in mainly astrogliosis in the corpus callosum without marked changes in the SVZ for GFAP, CDllb, or lymphocyte infiltration (Figures 7(c), 7(d), and 7(e)). At 2 weeks posttransplantation, HEK-Tom cells induced a robust, localized reaction in astrocytes (Figure $7(\mathrm{f})$ ), macrophages/microglia cells (Figure $7(\mathrm{~g})$ ), and T-lymphocytes (Figure 7(h)). Finally, increased Gli1-YFP labeling was observed in the SVZ adjacent to HEK-Tom cells (Figures 7(i) and $7(\mathrm{j})$ ). Thus, the robust immune rejection response to human cells activated Shh signaling in endogenous cells in the adjacent regions of the SVZ.

\section{Discussion}

NSCs hold therapeutic potential for cell replacement and/or for modulatory interactions with endogenous cells to promote regenerative processes. The current study examined effects of intraventricular transplantation of adult NSCs in experimental TBI. NSCs were transplanted at 2 weeks post-TBI to reflect a clinical time point that could be practical for clinical care decisions and for preparation of immunologically compatible cells for transplantation. Adult NSCs were used as a stem cell source with a low risk of tumor formation. A relatively low number of cells were transplanted to minimize mass effects and address the limited amplification capacity that may be likely to occur using protocols to generate autologous NSCs. 
Gli1 $^{\text {CreERT(2) }}{ }^{\text {R26tdTomato host mice }}$

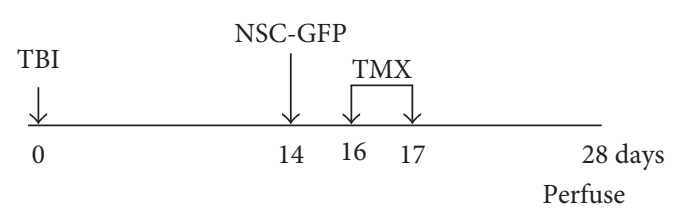

(a)

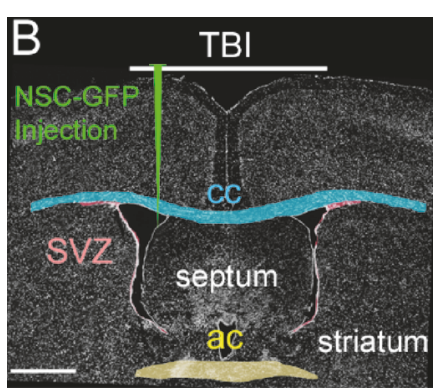

(b)

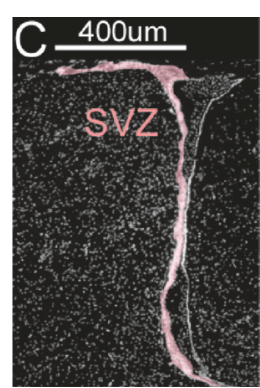

(c)

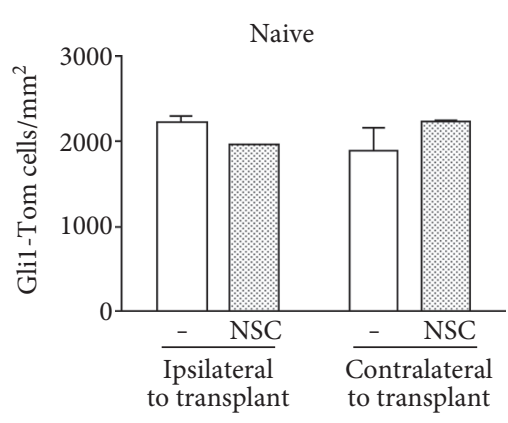

(d)

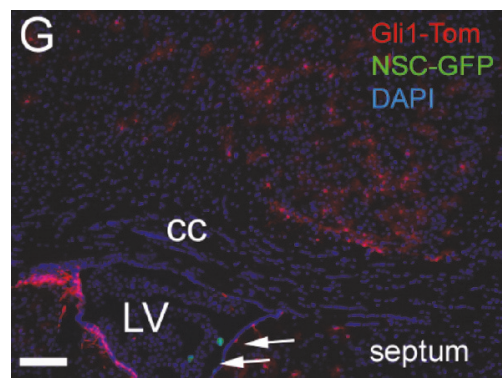

(g)

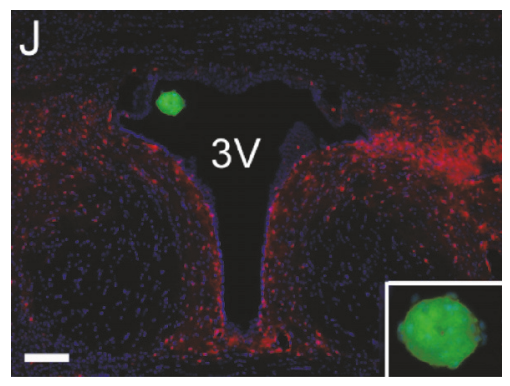

(j)

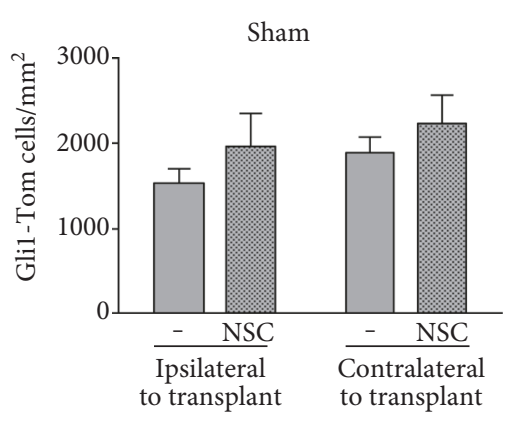

(e)

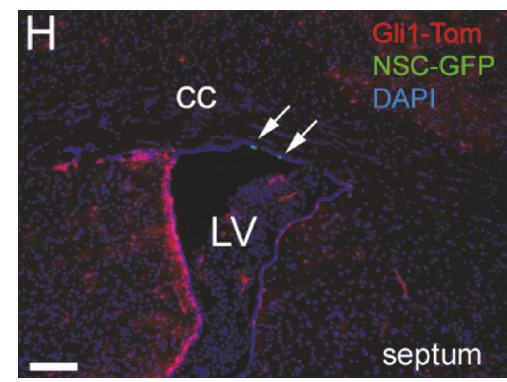

(h)

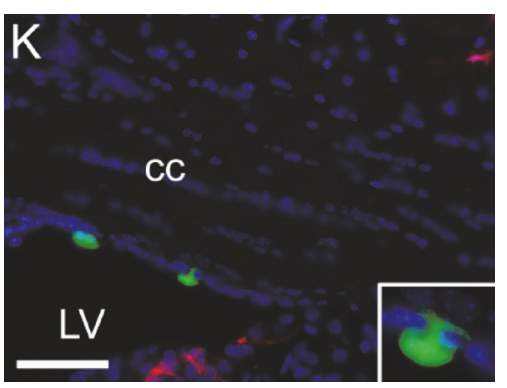

(k)

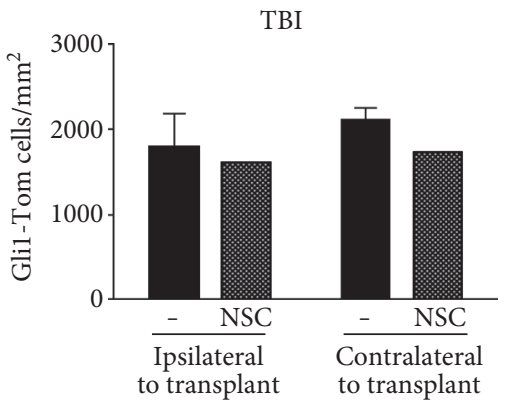

(f)

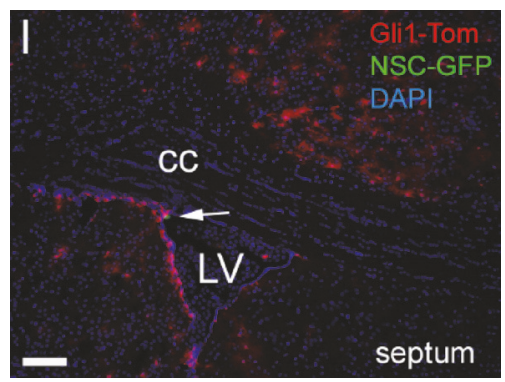

(i)

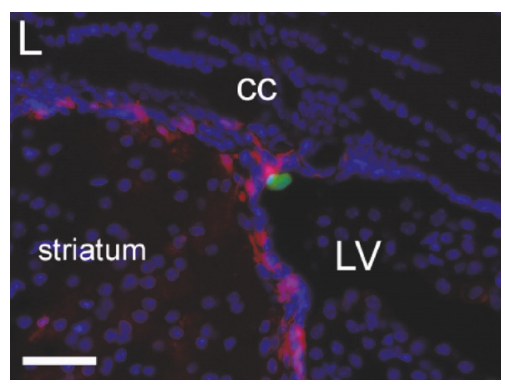

(1)

FIGURE 5: Induced genetic in vivo labeling of host cells expressing Gli1 in the subventricular zone (SVZ) following traumatic brain injury (TBI) and NSC transplantation. Timeline for $\mathrm{Gli1}^{\mathrm{CreERT2}} ;$ R26tdTomato host mice (a) with diagrams of TBI and injection site (b) and the SVZ region quantified (c) in naïve, sham, or TBI conditions. NSC-GFP cells for transplantation were isolated from mice constitutively expressing green fluorescent protein. (d-f) Quantification of cells actively responding to canonical Shh signaling (Gli1-Tom cells), in the SVZ showed no significant change in naïve (d), sham (e), or TBI (f) mice following injection of NSC-GFP as compared to vehicle. Low magnification images of the full SVZ regions are provided in Figure S4. (g-l) NSC-GFP cells transplanted into the lateral ventricle (LV) nestled within the choroid plexus (g) or adhered to the walls of the lateral ventricles (h, i) and were also found within the third ventricle (j). Gli1-Tom-labeled cells are not evident in the corpus callosum (cc). In contrast, robust Gli1-Tom label cells are found in the SVZ and in the cortex. Higher magnification of cells shown at the arrows (h, i). One cell has processes extended around the ependymal cell layer ((k), inset). Scale bars $=1 \mathrm{~mm}(\mathrm{~b}), 400 \mu \mathrm{m}(\mathrm{c}), 200 \mu \mathrm{m}(\mathrm{g}-\mathrm{j}), 50 \mu \mathrm{m}(\mathrm{k}, \mathrm{l})$. Quantification included cohorts of naïve + vehicle $(n=3)$, naïve + NSC-GFP $(n=2)$, sham + vehicle $(n=4)$, sham + NSC-GFP $(n=4)$, TBI + vehicle $(n=3)$, and TBI + NSCGFP $(n=4)$. 


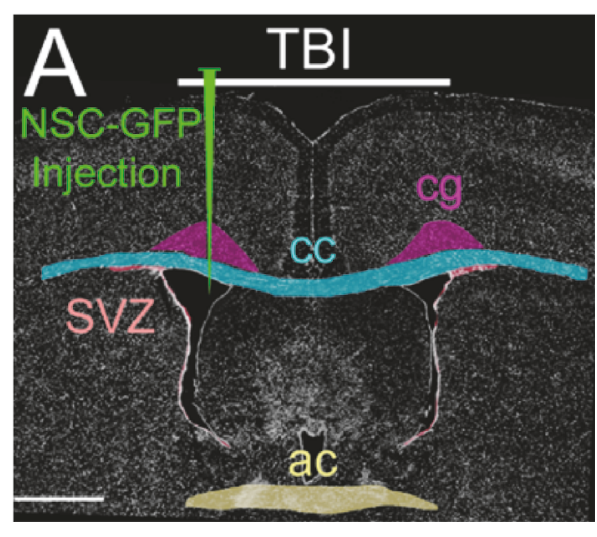

(a)

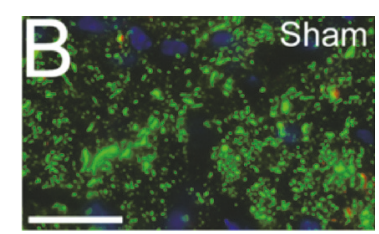

(b)

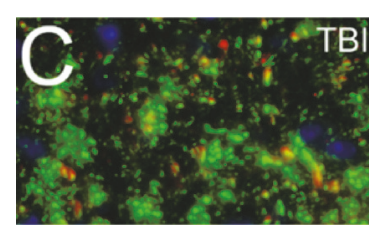

(c)

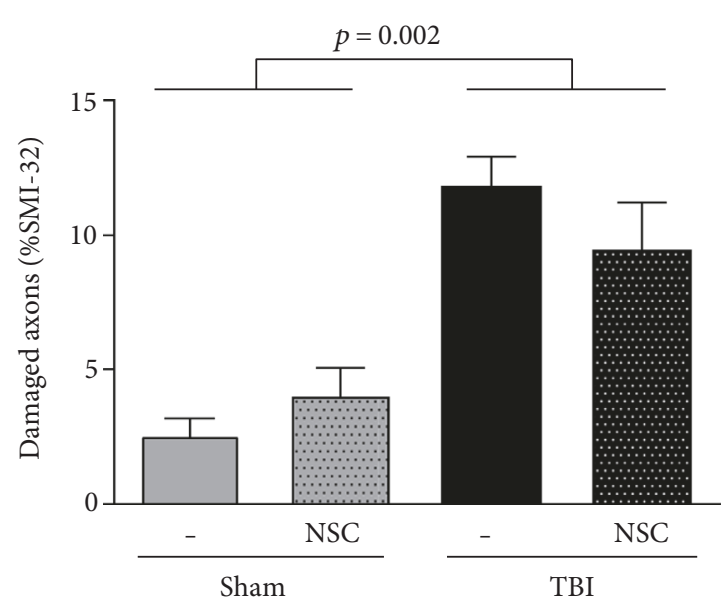

(d)

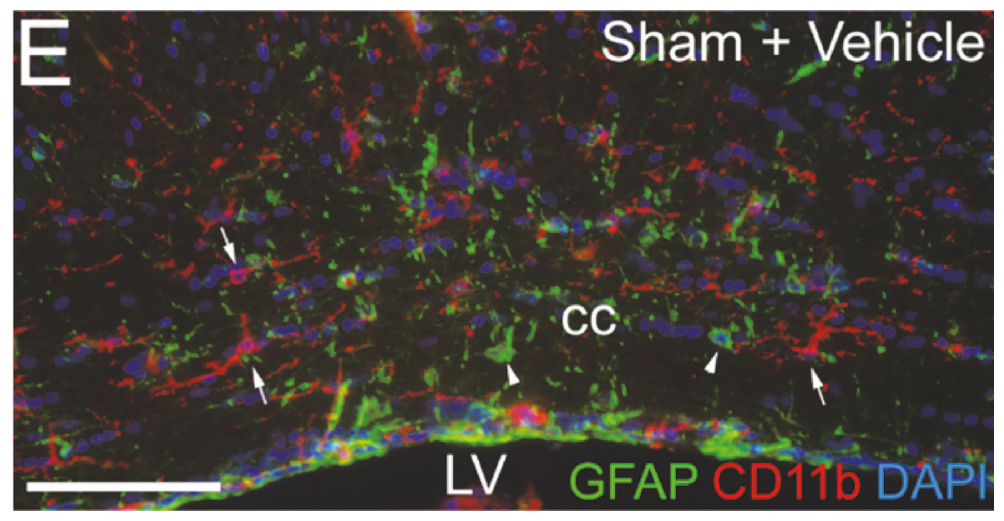

(e)

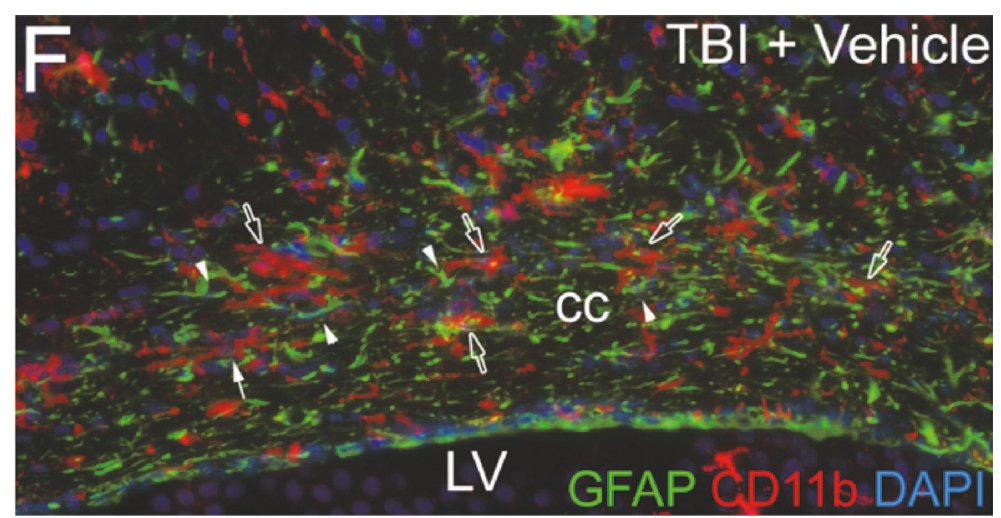

(f)

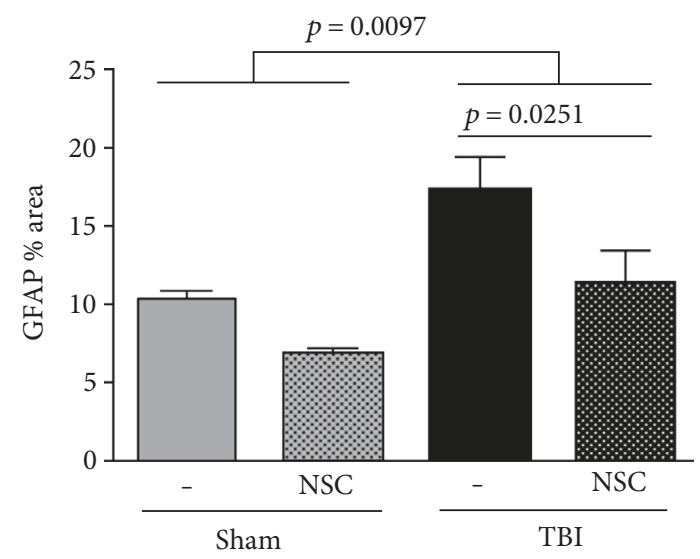

$\left(e^{\prime}\right)$

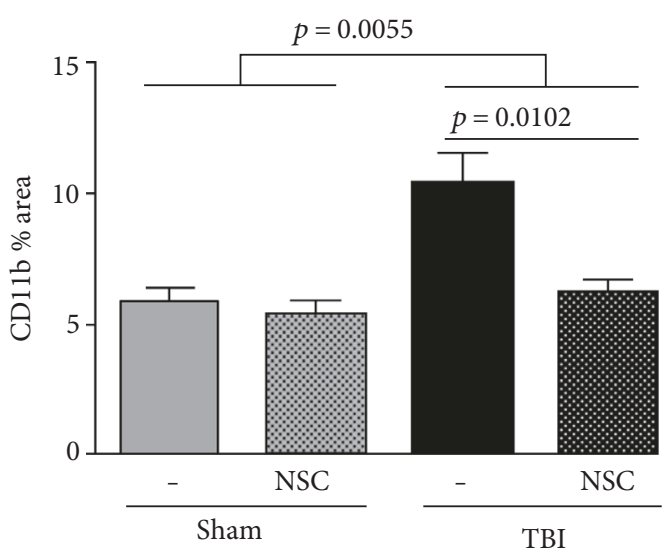

(f)

Figure 6: Continued. 


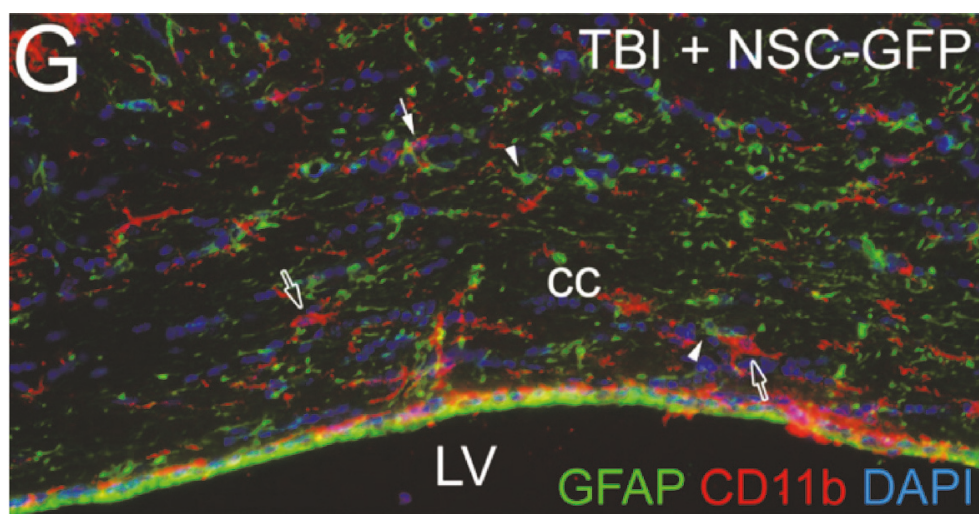

(g)

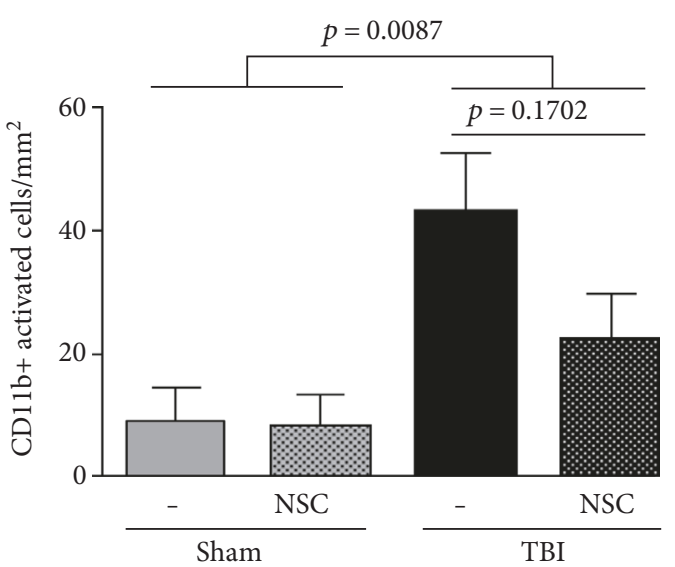

$\left(\mathrm{g}^{\prime}\right)$

FIGURE 6: NSC transplantation into the lateral ventricle (LV) following traumatic brain injury (TBI) suppresses injury-induced neuroinflammation in the corpus callosum. (a) Experimental diagram showing corpus callosum (cc) and cingulum regions (cg) quantified at the coronal level of the anterior commissure (ac) for mice prepared as detailed in Figures 5 and S4. Mice were analyzed at two weeks after NSC/vehicle injection which was four weeks after TBI/sham surgery. (b-d) Axons in the cingulum cut through transversely exhibit immunoreactivity for total neurofilaments (green) or SMI-32 (red) to detect nonphosphorylated neurofilaments as an indicator of axon damage. SMI-32-labeled axons are significantly increased in TBI, as compared to sham mice (d). (e-g) Coronal sections of the corpus callosum immunostained for GFAP to detect astrocytes (green, arrowheads) and Cd11b to identify microglia/macrophages (red, arrows) with DAPI nuclear stain (blue). As compared to sham mice (e), immunoreactivity for reactive changes in GFAP and CDllb is evident after TBI (f) and attenuated in TBI mice with NSC transplantation (g). (e', f, and g') Quantification of neuroinflammation in the corpus callosum. Immunoreactivity for GFAP (e') and CD11b ( $f$, g') was significantly increased in mice with TBI. NSC-GFP transplantation following TBI significantly reduced GFAP (e') and CD11b (f) immunoreactivity. Scale bar $=1 \mathrm{~mm}(\mathrm{a}), 20 \mu \mathrm{m}(\mathrm{b}), 100 \mu \mathrm{m}$ (e). Quantification included cohorts of sham + vehicle $(n=3)$, sham + NSC-GFP $(n=3)$, TBI + vehicle $(n=3)$, TBI + NSC-GFP $(n=3$ for neurofilaments; $n=4$ for CDllb GFAP).

Our studies focused on neuroinflammation and neuroregeneration associated with damage in white matter tracts from diffuse axonal injury in TBI. NSCs transplanted into the lateral ventricles survived and remained undifferentiated morphologically as single cells, or small spheres, adhered along the ventricle wall and nestled in the choroid plexus. NSC transplantation had a significant immunomodulatory effect in reducing astrogliosis and microglial/macrophage activation in the corpus callosum after TBI.

Transgenic mouse reporter lines were used to monitor in vivo activation of the Shh pathway as a candidate mechanism for transplanted NSC regulation of neuroinflammation as well as neuroregeneration from endogenous stem and progenitor cell populations. Genetic labeling of cells transcribing Shh identified neurons as the predominant source of the Shh ligand in the host tissues, which is consistent with prior reports of Shh expression [9, 32-34]. Shh labeling was not noticeably altered by TBI or NSC transplantation. TBI did not induce $S h h$ expression in reactive astrocytes, activated microglia, or other cells within the corpus callosum.

Using NSCs isolated from transgenic reporter mice, we showed that a subset of transplanted NSCs expressed Shh in vivo. Interestingly, Shh expression was suppressed in NSCs after TBI, but only among cells in the lateral ventricle region, indicating a specific NSC response based on proximity to the injury. In complementary studies, transgenic reporter mice were used as hosts with genetic labeling of cells transcribing Gli1, which is a readout of an active signaling through the canonical Shh pathway. Endogenous cells in the host SVZ exhibited robust Gli1 labeling. However, NSC transplantation did not activate Gli1 expression in either the SVZ or in the corpus callosum.

White matter tracts adjacent to the lateral ventricles have been shown to benefit from the effects of intraventricular cell transplantation in experimental models of multiple sclerosis. Neural stem/precursor cell intraventricular transplantation promoted remyelination in experimental autoimmune encephalomyelitis, which produces focal demyelinating lesions in multiple sites throughout the brain and spinal cord [35]. Intraventricular transplantation of syngeneic NSCs reduced astroglial scarring, which may be mediated through downregulation of factors in the lesion environment that drive reactive astrogliosis, such as fibroblast growth factor [35]. Other studies in this model also showed that neural stem/precursor cells transplanted into the ventricle acted through an anti-inflammatory mechanism to reduce both the innate and adaptive immune response [36]. The use of syngeneic NSCs in these reports, as in the current studies, indicates that immunosuppression is an effect of adult NSC characteristics and is not likely due to factors associated with a general rejection response to the transplanted cells. Using the cuprizone model of corpus callosum demyelination, intraventricular transplantation of NSCs isolated from newborn mice was shown to promote endogenous cell remyelination, which may be mediated through NSC secretion of platelet-derived growth factor and fibroblast growth factor 2 that act as mitogens for oligodendrocyte progenitors [12]. These findings indicate the potential for intraventricular 
Gli1 $^{\operatorname{CreERT(2)} ; R 26 Y F P}$ host mice
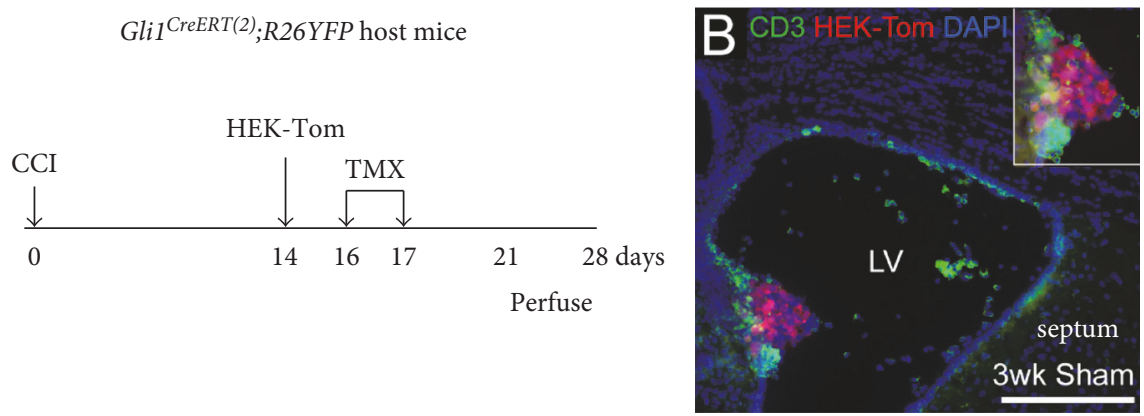

(a)

(b)

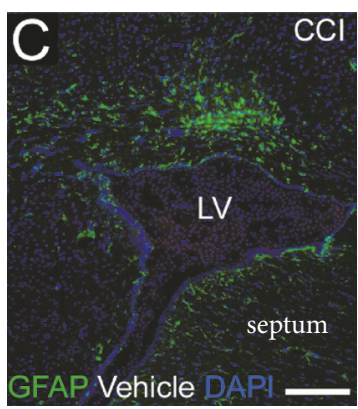

(c)

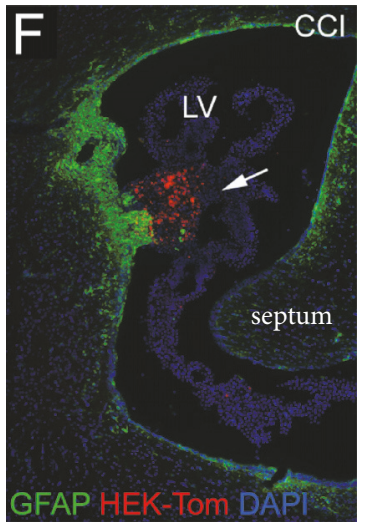

(f)

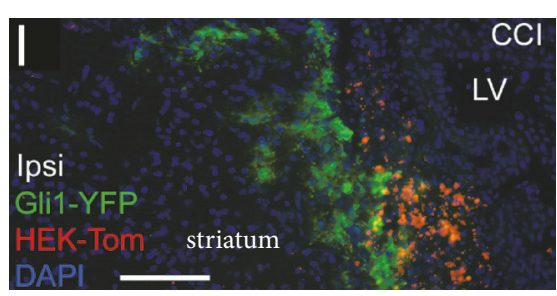

(i)

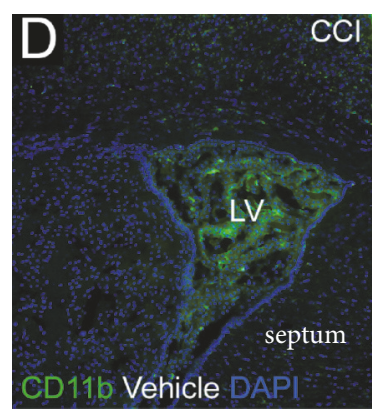

(d)

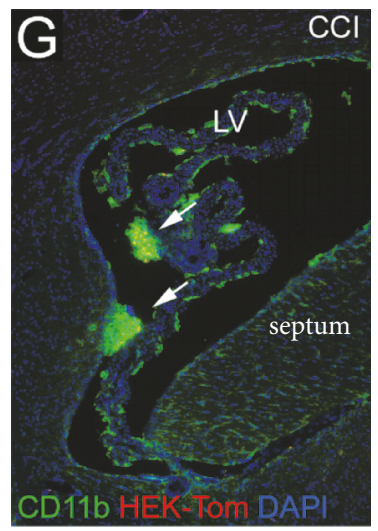

(g)

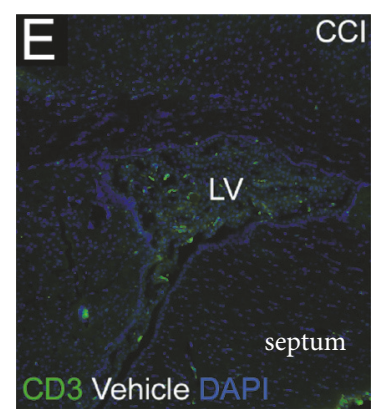

(e)

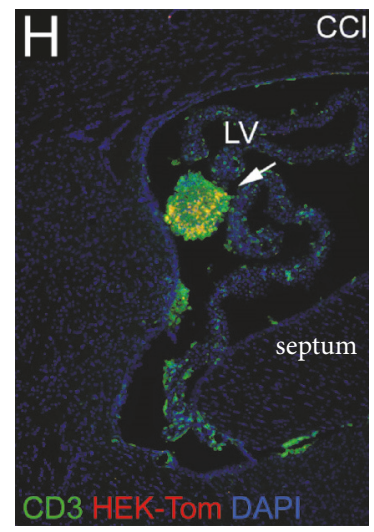

(h)

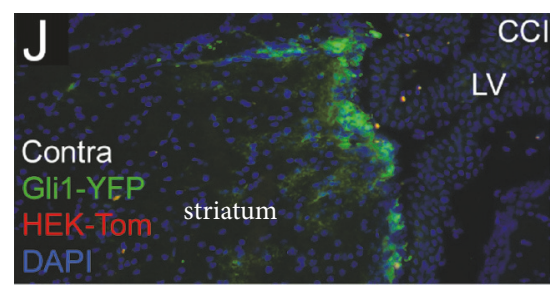

(j)

FIGURE 7: Induced genetic in vivo labeling of host cells expressing Gli1 in the subventricular zone (SVZ) following brain injury and human non-NSC transplantation. (a) Experimental time line for human HEK transplantation into Gli1 ${ }^{\text {CreERT2 }}{ }_{\text {; R26YFP host mice. The HEK293 cells }}$ expressing the tdTomato reporter (HEK-Tom cells) were transplanted into the lateral ventricle (LV) 2 weeks after craniotomy and the controlled cortical impact (CCI) model of TBI to produce cortical tissue damage. (b) Immunohistochemistry for the pan T-lymphocyte cell (T-cell) marker CD3 shows T-lymphocytes infiltrate regions surrounding the human HEK293 cells as early as 1 week following transplantation into a sham mouse. (c-h) Coronal sections from injured mice were immunostained with either GFAP (astrocytes, green), CD11b (macrophages, green), or CD3 (T-cells, green) to analyze the host immune response at 2 weeks postinjection. Immunolabeling for GFAP (c, f), CD11b (d, g), and CD3 (e, h) shows a localized immune response to HEK-Tom cells in the LV (f-h) that is not observed with vehicle injection (c-e). (i-j) Genetic in vivo labeling of host cells with YFP (green) indicates Gli1 expression after HEK-Tom transplantation. Gli1-YFP labeling is increased in the ipsilateral SVZ adjacent to HEK-Tom cells (i) compared to the contralateral side (j). All sections were counterstained with DAPI (blue) nuclear stain. Scale bar $=200 \mu \mathrm{m}$ (c), (d), $100 \mu \mathrm{m}$ (i). Representative images are shown from analysis of sham + HEK-Tom $(n=1)$, CCI + vehicle $(n=1)$, CCI + HEK-Tom $(n=2)$. 
delivery of neural stem/precursor cells to both modulate the immune response and support regenerative mechanisms after white matter injury. Results of the current study are consistent with NSCs suppressing the immune response, but do not provide evidence of transplanted NSC stimulation of a regenerative response in endogenous cells of the host SVZ.

NSCs transplanted into the ventricles may act through direct and indirect mechanisms to modulate expression of regulatory molecules. NSCs can synthesize growth factors in vivo after transplantation, as shown for in vivo synthesis of Shh in transplanted NSCs in the current experiments with genetic in vivo labeling induced from the Shh promoter. Transplanted NSCs may also interact with host cells to modulate expression of signaling from endogenous cells of the choroid plexus, ventricle wall, and/or SVZ. However, transplanted NSCs did not appear to induce Shh expression in host ependymal or choroid plexus cells in our experiments.

To further explore host-transplant interactions, intraventricular transplantation of human non-NSC cells was used as a positive control to elicit an immune rejection response to then examine the effect on Gli1 labeling in the host SVZ. Tlymphocytes and macrophages responded strongly to HEK cells transplanted after an invasive form of TBI. Interestingly, this inflammatory response corresponded with localized Gli1 labeling in adjacent host SVZ cells. Transplanted HEK cells, but not NSCs, also disrupted the normal expression of syndecan-1 in the choroid plexus (data not shown), which indicates that HEK cells disrupt the integrity of the bloodcerebrospinal fluid barrier at the choroid plexus that normally prevents immune cell infiltration [37]. Therefore, T-lymphocytes or macrophage activation may be required to induce active Shh signaling. Breakdown of the barriers between the blood and cerebrospinal fluid or brain compartments may also contribute. Consistent with this interpretation, apoptotic/stimulated T-lymphocytes have been reported to release microvesicles containing Shh [38]. In addition, Shh signaling may regulate the integrity of the blood-brain barrier in that inflammation stimulates increased Shh immunolabeling of astrocytes that interact with Gli1 expressing endothelial cells [39]. Overall, our current observations are consistent with previous studies that proposed blood-brain barrier disruption may be required to stimulate Shh signaling in the adult brain, while astrogliosis alone is not sufficient $[25,33]$. However, it is not clear yet how Gli1 activation may be influenced by blood-brain barrier disruption. Gli1 genetic labeling in astrocytes was reduced in lesion areas of the cerebral cortex when TMX was administered after TBI from controlled cortical impact, which results in bleeding from vascular injury [17].

Prior studies from our lab and others have reported a lack of Gli1 expression in the corpus callosum of naïve adult mice and after TBI $[9,17]$. Gli1 genetic labeling of cells in the corpus callosum was not observed even after microinjection of a Shh pathway agonist into the corpus callosum in naïve adult mice [17]. Indeed, recent studies showed that inhibition of Gli1 may be required for NSCs to be recruited from the SVZ to demyelinated lesions and to subsequently differentiate into oligodendrocytes [40]. This interpretation is also consistent with our prior study showing early transient downregulation of Gli1 in vivo labeling in the SVZ in a model of TBI that produces axon damage and demyelination in the corpus callosum $[17,18]$. In contrast, studies that used in situ hybridization to detect Gli1 expression reported an increase of Gli1 transcripts in corpus callosum lesions after experimental demyelination [13]. The conflicting results could be due to differences in the approaches used to detect Gli1, but also to differences in the inflammatory signals and bloodbrain barrier integrity associated with the different pathologies. In addition, although the Gli1 genetic labeling approach used in the current study is a specific indicator of active in vivo signaling through the canonical Shh pathway, Shh can also act through noncanonical mechanisms that do not require Gli1 activation $[41,42]$.

\section{Conclusions}

These studies demonstrate that adult NSCs transplanted into the lateral ventricle remain within the lateral and third ventricles for at least two weeks and significantly reduce neuroinflammation in the corpus callosum. These findings are consistent with potential applications for the diffuse white matter injury experienced in TBI. We report that NSC transplantation significantly reduces reactive astrogliosis and microglial/macrophage activation in the corpus callosum after TBI. These studies are also the first to investigate the potential for transplanted NSCs to act through Shh signaling using induced genetic in vivo labeling. This genetic approach demonstrated Shh ligand expression in vivo in a subset of transplanted NSCs, which was suppressed by TBI. A robust Gli1 response to Shh signaling in endogenous cells of the host SVZ was observed in adult mice. However, NSC transplantation did not stimulate Shh-mediated activation of a regenerative response in the host SVZ. And, NSC reduction of neuroinflammation in the corpus callosum after TBI did not involve Shh signaling through Gli1. Further studies are warranted to better understand the therapeutic potential of transplanted NSCs for suppressing neuroinflammation. Such studies would need to extend to severe forms of TBI and at later time points after injury that will be important for clinical translation and evaluation of the risk benefit of cell transplantation as a therapeutic intervention.

\section{Conflicts of Interest}

The authors declare that there is no conflict of interest regarding the publication of this paper.

\section{Acknowledgments}

This work was funded by the Department of Defense in the Center for Neuroscience and Regenerative Medicine (CNRM). The authors appreciate the technical assistance of Tuan Le and Laurel Beer. The authors thank Dr. Sohyun Ahn (National Institute for Child Health, Bethesda, MD) for providing the Gli1 ${ }^{\text {CreERT2 }}$;R26YFP and Gli1 ${ }^{\text {CreERT2 }}$;R26tdTomato mice and Dr. Sharon Juliano for providing the C57BL/6-Tg(UBC-GFP)30Scha/J mice. 


\section{References}

[1] B. Roozenbeek, A. I. Maas, and D. K. Menon, "Changing patterns in the epidemiology of traumatic brain injury," Nature Reviews. Neurology, vol. 9, no. 4, pp. 231-236, 2013.

[2] D. H. Smith, R. Hicks, and J. T. Povlishock, "Therapy development for diffuse axonal injury," Journal of Neurotrauma, vol. 30, no. 5, pp. 307-323, 2013.

[3] A. W. Selassie, E. Zaloshnja, J. A. Langlois, T. Miller, P. Jones, and C. Steiner, "Incidence of long-term disability following traumatic brain injury hospitalization, United States, 2003," The Journal of Head Trauma Rehabilitation, vol. 23, no. 2, pp. 123-131, 2008.

[4] C. S. Hill, M. P. Coleman, and D. K. Menon, "Traumatic axonal injury: mechanisms and translational opportunities," Trends in Neurosciences, vol. 39, no. 5, pp. 311-324, 2016.

[5] S. Margulies, G. Anderson, F. Atif et al., "Combination therapies for traumatic brain injury: retrospective considerations," Journal of Neurotrauma, vol. 33, no. 1, pp. 101-112, 2016.

[6] D. Drago, C. Cossetti, N. Iraci et al., "The stem cell secretome and its role in brain repair," Biochimie, vol. 95, no. 12, pp. 2271-2285, 2013.

[7] F. Doetsch, I. Caille, D. A. Lim, J. M. García-Verdugo, and A. Alvarez-Buylla, "Subventricular zone astrocytes are neural stem cells in the adult mammalian brain," Cell, vol. 97, no. 6, pp. 703-716, 1999.

[8] V. Palma, D. A. Lim, N. Dahmane et al., "Sonic hedgehog controls stem cell behavior in the postnatal and adult brain," Development, vol. 132, no. 2, pp. 335-344, 2005.

[9] A. D. Garcia, R. Petrova, L. Eng, and A. L. Joyner, "Sonic hedgehog regulates discrete populations of astrocytes in the adult mouse forebrain," The Journal of Neuroscience, vol. 30, no. 41, pp. 13597-13608, 2010.

[10] R. Petrova, A. D. Garcia, and A. L. Joyner, "Titration of GLI3 repressor activity by sonic hedgehog signaling is critical for maintaining multiple adult neural stem cell and astrocyte functions," The Journal of Neuroscience, vol. 33, no. 44, pp. 17490-17505, 2013.

[11] V. F. Rafuse, P. Soundararajan, C. Leopold, and H. A. Robertson, "Neuroprotective properties of cultured neural progenitor cells are associated with the production of sonic hedgehog," Neuroscience, vol. 131, no. 4, pp. 899-916, 2005.

[12] O. Einstein, Y. Friedman-Levi, N. Grigoriadis, and T. Ben-Hur, "Transplanted neural precursors enhance host brain-derived myelin regeneration," The Journal of Neuroscience, vol. 29, no. 50, pp. 15694-15702, 2009.

[13] J. Ferent, C. Zimmer, P. Durbec, M. Ruat, and E. Traiffort, "Sonic hedgehog signaling is a positive oligodendrocyte regulator during demyelination," The Journal of Neuroscience, vol. 33, no. 5, pp. 1759-1772, 2013.

[14] M. Donega, E. Giusto, C. Cossetti, J. Schaeffer, and S. Pluchino, "Systemic injection of neural stem/progenitor cells in mice with chronic EAE," Journal of Visualized Experiments, vol. 86, 2014.

[15] E. Giusto, M. Donegà, C. Cossetti, and S. Pluchino, "Neuroimmune interactions of neural stem cell transplants: from animal disease models to human trials," Experimental Neurology, vol. 260, pp. 19-32, 2014.

[16] G. M. Sullivan, A. J. Mierzwa, N. Kijpaisalratana et al., "Oligodendrocyte lineage and subventricular zone response to traumatic axonal injury in the corpus callosum," Journal of
Neuropathology and Experimental Neurology, vol. 72, no. 12, pp. 1106-1125, 2013.

[17] A. J. Mierzwa, G. M. Sullivan, L. A. Beer, S. Ahn, and R. C. Armstrong, "Comparison of cortical and white matter traumatic brain injury models reveals differential effects in the subventricular zone and divergent sonic hedgehog signaling pathways in neuroblasts and oligodendrocyte progenitors," ASN Neuro, vol. 6, no. 5, 2014.

[18] A. J. Mierzwa, C. M. Marion, G. M. Sullivan, M. D. DP, and R. C. Armstrong, "Components of myelin damage and repair in the progression of white matter pathology after mild traumatic brain injury," Journal of Neuropathology and Experimental Neurology, vol. 74, no. 3, pp. 218-232, 2015.

[19] B. D. Harfe, P. J. Scherz, S. Nissim, H. Tian, M. M. AP, and C. J. Tabin, "Evidence for an expansion-based temporal Shh gradient in specifying vertebrate digit identities," Cell, vol. 118, no. 4, pp. 517-528, 2004.

[20] S. Ahn and A. L. Joyner, "Dynamic changes in the response of cells to positive hedgehog signaling during mouse limb patterning," Cell, vol. 118, no. 4, pp. 505-516, 2004.

[21] R. A. Ihrie, J. K. Shah, C. C. Harwell et al., "Persistent sonic hedgehog signaling in adult brain determines neural stem cell positional identity," Neuron, vol. 71, no. 2, pp. 250-262, 2011.

[22] S. Ahn and A. L. Joyner, "In vivo analysis of quiescent adult neural stem cells responding to sonic hedgehog," Nature, vol. 437, no. 7060, pp. 894-897, 2005.

[23] S. Srinivas, T. Watanabe, C. S. Lin et al., "Cre reporter strains produced by targeted insertion of EYFP and ECFP into the ROSA26 locus,” BMC Developmental Biology, vol. 1, p. 4, 2001.

[24] L. Madisen, T. A. Zwingman, S. M. Sunkin et al., "A robust and high-throughput Cre reporting and characterization system for the whole mouse brain," Nature Neuroscience, vol. 13, no. 1, pp. 133-140, 2010.

[25] M. D. Muzumdar, B. Tasic, K. Miyamichi, L. Li, and L. Luo, "A global double-fluorescent Cre reporter mouse," Genesis, vol. 45, no. 9, pp. 593-605, 2007.

[26] Q. Zhou, C. L. Dalgard, C. Wynder, and M. L. Doughty, "Valproic acid inhibits neurosphere formation by adult subventricular cells by a lithium-sensitive mechanism," Neuroscience Letters, vol. 500, no. 3, pp. 202-206, 2011.

[27] Q. Zhou, C. L. Dalgard, C. Wynder, and M. L. Doughty, "Histone deacetylase inhibitors SAHA and sodium butyrate block G1-to-S cell cycle progression in neurosphere formation by adult subventricular cells," BMC Neuroscience, vol. 12, p. $50,2011$.

[28] R. C. Armstrong, T. Q. Le, E. E. Frost, R. C. Borke, and A. C. Vana, "Absence of fibroblast growth factor 2 promotes oligodendroglial repopulation of demyelinated white matter," The Journal of Neuroscience, vol. 22, no. 19, pp. 8574-8585, 2002.

[29] N. R. Dobson, R. T. Moore, J. E. Tobin, and R. C. Armstrong, "Leukemia/lymphoma-related factor regulates oligodendrocyte lineage cell differentiation in developing white matter," Glia, vol. 60, no. 9, pp. 1378-1390, 2012.

[30] B. D. Trapp, J. Peterson, R. M. Ransohoff, R. Rudick, S. Mörk, and L. Bö, "Axonal transection in the lesions of multiple sclerosis," The New England Journal of Medicine, vol. 338, no. 5, pp. 278-285, 1998.

[31] A. Alvarez-Buylla and R. A. Ihrie, "Sonic hedgehog signaling in the postnatal brain," Seminars in Cell \& Developmental Biology, vol. 33, pp. 105-111, 2014. 
[32] C. C. Harwell, P. R. Parker, S. M. Gee et al., "Sonic hedgehog expression in corticofugal projection neurons directs cortical microcircuit formation," Neuron, vol. 73, no. 6, pp. 11161126, 2012.

[33] S. Sirko, G. Behrendt, P. A. Johansson et al., "Reactive glia in the injured brain acquire stem cell properties in response to sonic hedgehog. [corrected]," Cell Stem Cell, vol. 12, no. 4, pp. 426-439, 2013.

[34] W. T. Farmer, T. Abrahamsson, S. Chierzi et al., "Neurons diversify astrocytes in the adult brain through sonic hedgehog signaling," Science, vol. 351, no. 6275, pp. 849-854, 2016.

[35] S. Pluchino, A. Quattrini, E. Brambilla et al., "Injection of adult neurospheres induces recovery in a chronic model of multiple sclerosis," Nature, vol. 422, no. 6933, pp. 688694, 2003.

[36] S. Pluchino, L. Zanotti, B. Rossi et al., "Neurosphere-derived multipotent precursors promote neuroprotection by an immunomodulatory mechanism," Nature, vol. 436, no. 7048, pp. 266-271, 2005.

[37] X. Zhang, C. Wu, J. Song, M. Götte, and L. Sorokin, "Syndecan-1, a cell surface proteoglycan, negatively regulates initial leukocyte recruitment to the brain across the choroid plexus in murine experimental autoimmune encephalomyelitis," Journal of Immunology, vol. 191, no. 9, pp. 4551-4561, 2013.

[38] M. C. Martinez, F. Larbret, F. Zobairi et al., "Transfer of differentiation signal by membrane microvesicles harboring hedgehog morphogens," Blood, vol. 108, no. 9, pp. 30123020, 2006.

[39] J. I. Alvarez, A. Dodelet-Devillers, H. Kebir et al., "The hedgehog pathway promotes blood-brain barrier integrity and CNS immune quiescence," Science, vol. 334, no. 6063, pp. 1727-1731, 2011.

[40] J. Samanta, E. M. Grund, H. M. Silva, J. J. Lafaille, G. Fishell, and J. L. Salzer, "Inhibition of Gli1 mobilizes endogenous neural stem cells for remyelination," Nature, vol. 526, no. 7573 , pp. 448-452, 2015.

[41] D. Brennan, X. Chen, L. Cheng, and N. A. Riobo, "Noncanonical hedgehog signaling," Vitamins and Hormones, vol. 88, pp. 55-72, 2012.

[42] D. J. Robbins, D. L. Fei, and N. A. Riobo, "The hedgehog signal transduction network," Science Signaling, vol. 5, no. 246, article re6, 2012. 

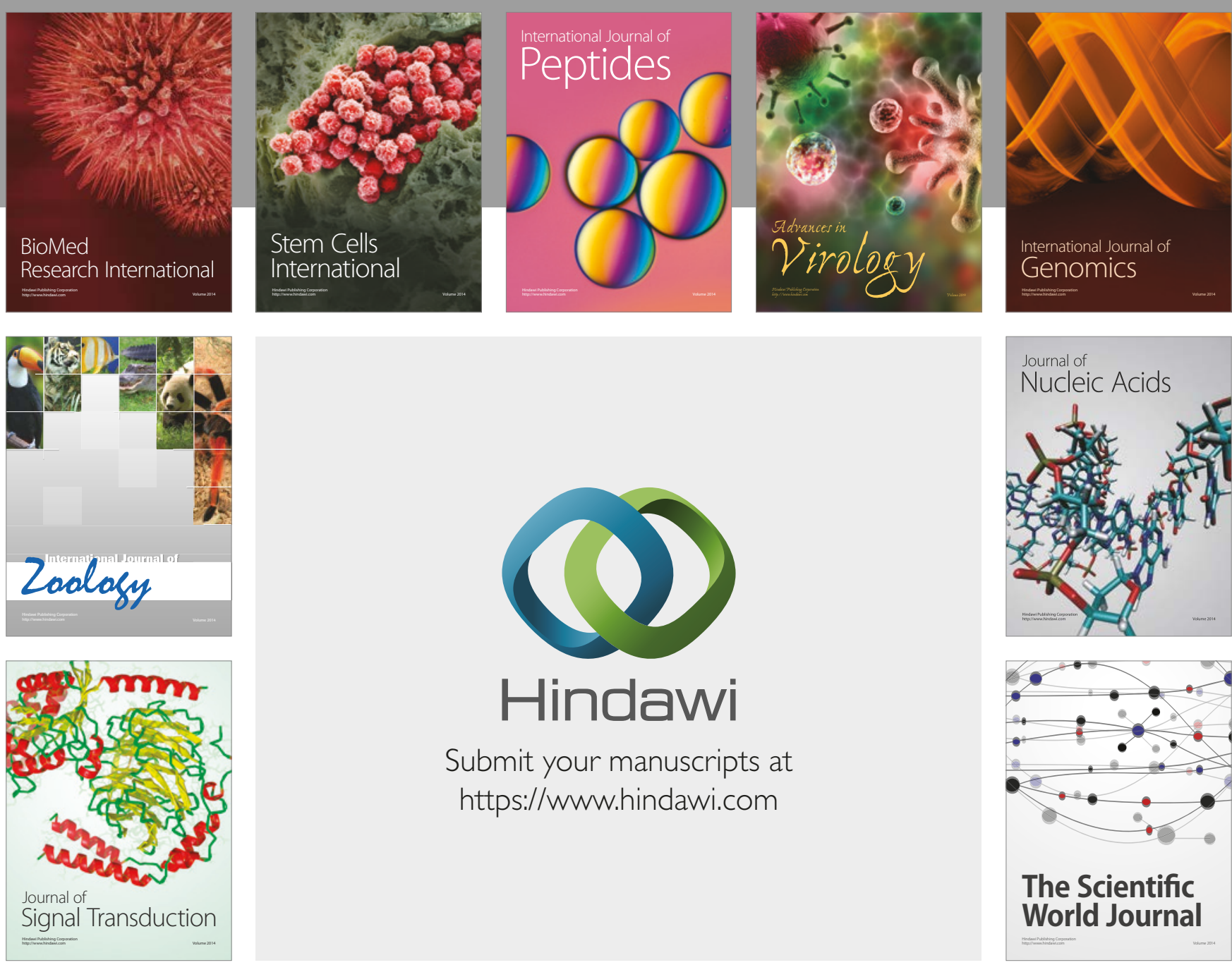

Submit your manuscripts at

https://www.hindawi.com
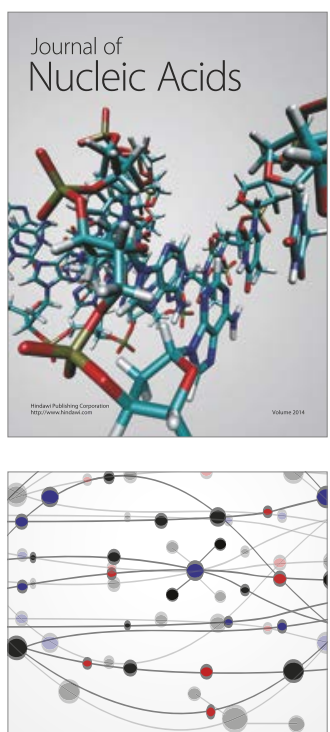

The Scientific World Journal

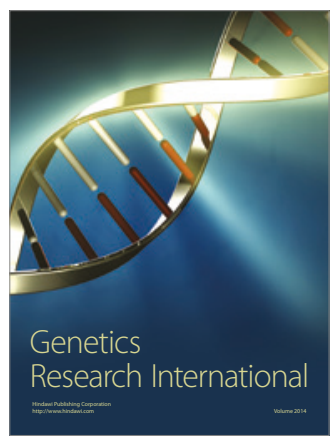

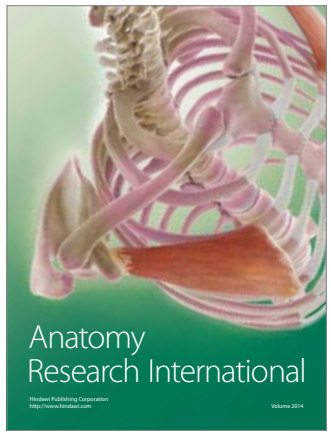

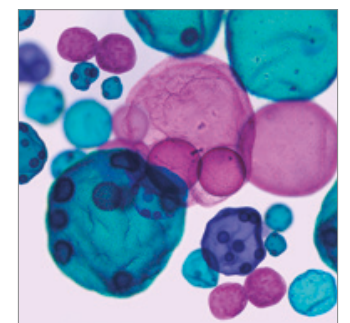

International Journal of Microbiology
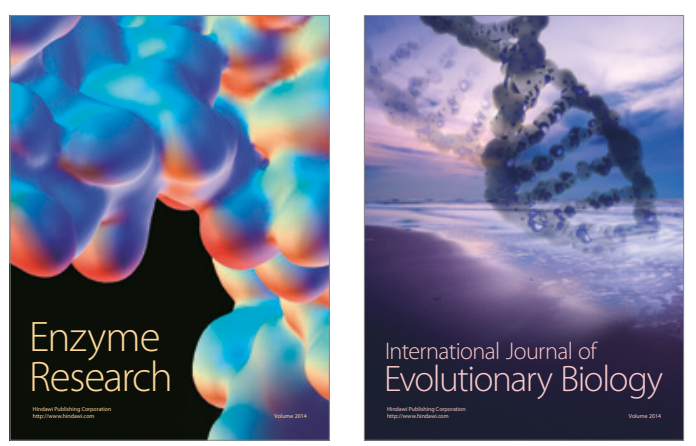
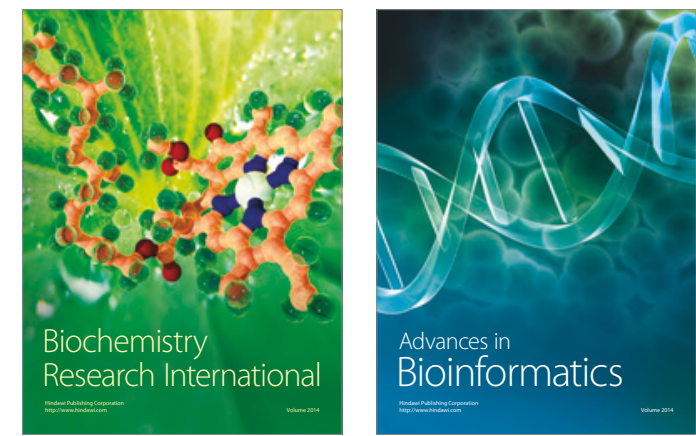

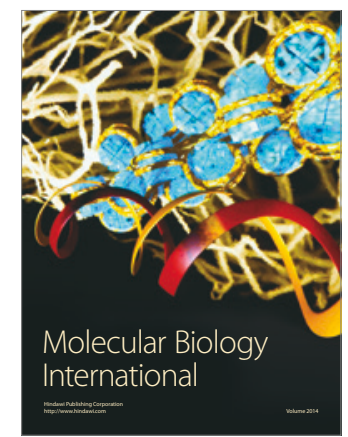

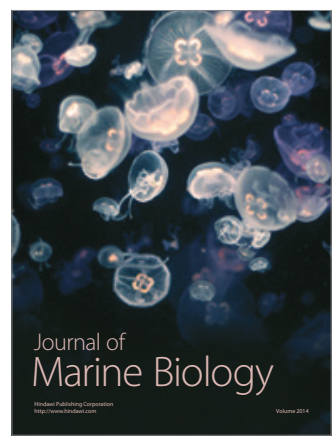

